\title{
A Survey of Alternating Permutations
}

\author{
Richard P. Stanley
}

\begin{abstract}
A permutation $a_{1} a_{2} \cdots a_{n}$ of $1,2, \ldots, n$ is alternating if $a_{1}>a_{2}<$ $a_{3}>a_{4}<\cdots$. We survey some aspects of the theory of alternating permutations, beginning with the famous result of Andre that if $E_{n}$ is the number of alternating permutations of $1,2, \ldots, n$, then $\sum_{n \geq 0} E_{n} \frac{x^{n}}{n !}=\sec x+\tan x$. Topics include refinements and $q$-analogues of $E_{n}$, various occurrences of $E_{n}$ in mathematics, longest alternating subsequences of permutations, umbral enumeration of special classes of alternating permutations, and the connection between alternating permutations and the $c d$-index of the symmetric group.
\end{abstract}

Dedicated to Reza Khosrovshahi on the occasion of his 70th birthday

\section{Basic enumerative properties.}

Let $\mathfrak{S}_{n}$ denote the symmetric group of all permutations of $[n]:=\{1,2, \ldots, n\}$. A permutation $w=a_{1} a_{2} \cdots a_{n} \in \mathfrak{S}_{n}$ is called alternating if $a_{1}>a_{2}<a_{3}>a_{4}<$ $\cdots$. In other words, $a_{i}<a_{i+1}$ for $i$ even, and $a_{i}>a_{i+1}$ for $i$ odd. Similarly $w$ is reverse alternating if $a_{1}\left\langle a_{2}>a_{3}<a_{4}>\cdots\right.$. (Some authors reverse these definitions.) Let $E_{n}$ denote the number of alternating permutations in $\mathfrak{S}_{n}$. (Set $E_{0}=1$.) For instance, $E_{4}=5$, corresponding to the permutations 2143, 3142, 3241,4132 , and 4231 . The number $E_{n}$ is called an Euler number because Euler considered the numbers $E_{2 n+1}$, though not with the combinatorial definition just given. (Rather, Euler defined them via equation (1.3) below.) The Euler numbers are not to be confused with the Eulerian numbers, which count permutations by number of descents. The involution

$$
a_{1} a_{2} \cdots a_{n} \mapsto n+1-a_{1}, n+1-a_{2}, \cdots, n+1-a_{n}
$$

on $\mathfrak{S}_{n}$ shows that $E_{n}$ is also the number of reverse alternating permutations in $\mathfrak{S}_{n}$. We write $\operatorname{Alt}_{n}$ (respectively, Ralt ${ }_{n}$ ) for the set of alternating (respectively, reverse alternating) permutations $w \in \mathfrak{S}_{n}$.

The subject of alternating permutations and Euler numbers has become so vast that it is impossible to give a comprehensive survey. We will confine ourselves to some highlights and to some special topics that we find especially interesting.

2000 Mathematics Subject Classification. Primary 05E10, Secondary 05E05.

This material is based upon work supported by the National Science Foundation under Grant No. 0604423. Any opinions, findings and conclusions or recommendations expressed in this material are those of the author and do not necessarily reflect those of the National Science Foundation. 
The fundamental enumerative property of alternating permutations is due to Desiré André [1] in 1879. (Note however that Ginsburg [35] asserts without giving a reference that Binet was aware before André that the coefficients of $\sec x$ count alternating permutations.)

THEOREM 1.1. We have

$$
\begin{aligned}
\sum_{n \geq 0} E_{n} \frac{x^{n}}{n !} & =\sec x+\tan x \\
& =1+x+\frac{x^{2}}{2 !}+2 \frac{x^{3}}{3 !}+5 \frac{x^{4}}{4 !}+16 \frac{x^{5}}{5 !}+61 \frac{x^{6}}{6 !}+272 \frac{x^{7}}{7 !}+1385 \frac{x^{8}}{8 !}+\cdots .
\end{aligned}
$$

Note that $\sec x$ is an even function (i.e, $\sec (-x)=\sec x$ ), while $\tan x$ is odd $(\tan (-x)=-\tan x)$. It follows from Theorem 1.1 that

$$
\begin{aligned}
\sum_{n \geq 0} E_{2 n} \frac{x^{2 n}}{(2 n) !} & =\sec x \\
\sum_{n \geq 0} E_{2 n+1} \frac{x^{2 n+1}}{(2 n+1) !} & =\tan x .
\end{aligned}
$$

For this reason $E_{2 n}$ is sometimes called a secant number and $E_{2 n+1}$ a tangent number.

We will sketch three proofs of Theorem 1.1.

First proof. Let $0 \leq k \leq n$. Choose a $k$-subset $S$ of $[n]=\{1,2, \ldots, n\}$ in $\left(\begin{array}{l}n \\ k\end{array}\right)$ ways, and set $\bar{S}=[n]-S$. Choose a reverse alternating permutation $u$ of $S$ in $E_{k}$ ways, and choose a reverse alternating permutation $v$ of $\bar{S}$ in $E_{n-k}$ ways. Let $w$ be the concatenation $u^{r}, n+1, v$, where $u^{r}$ denotes the reverse of $u$ (i.e., if $u=u_{1} \cdots u_{k}$ then $\left.u^{r}=u_{k} \cdots u_{1}\right)$. When $n \geq 2$, we obtain in this way every alternating and every reverse alternating permutation $w$ exactly once. Since there is a bijection between alternating and reverse alternating permutations of any finite (ordered) set, the number of $w$ obtained is $2 E_{n+1}$. Hence

$$
2 E_{n+1}=\sum_{k=0}^{n}\left(\begin{array}{l}
n \\
k
\end{array}\right) E_{k} E_{n-k}, \quad n \geq 1 .
$$

Set $y=\sum_{n>0} E_{n} x^{n} / n$ !. Taking into account the initial conditions $E_{0}=E_{1}=1$, equation (1.4) becomes the differential equation

$$
2 y^{\prime}=y^{2}+1, \quad y(0)=1 \text {. }
$$

The unique solution is $y=\sec x+\tan x$.

NotE. The clever counting of both alternating and reverse alternating permutations in the proof of Theorem 1.1 can be avoided at the cost of a little elegance. Namely, by considering the position of 1 in an alternating permutation $w$, we obtain the recurrence

$$
E_{n+1}=\sum_{\substack{1 \leq j \leq n \\
j \text { odd }}}\left(\begin{array}{l}
n \\
j
\end{array}\right) E_{j} E_{n-j}, \quad n \geq 1 .
$$

This recurrence leads to a system of differential equations for the power series $\sum_{n \geq 0} E_{2 n} x^{2 n} /(2 n)$ ! and $\sum_{n \geq 0} E_{2 n+1} x^{2 n+1} /(2 n+1)$ !. 
Second proof. For simplicity we consider only $E_{2 n}$. A similar, though somewhat more complicated, argument works for $E_{2 n+1}$. We want to show that

$$
\left(\sum_{n \geq 0} E_{2 n} \frac{x^{n}}{n !}\right)\left(1-\frac{x^{2}}{2 !}+\frac{x^{4}}{4 !}-\cdots\right)=1 .
$$

Equating coefficients of $x^{2 n} /(2 n)$ ! on both sides gives

$$
E_{2 n}=\left(\begin{array}{l}
n \\
2
\end{array}\right) E_{2 n-2}-\left(\begin{array}{l}
n \\
4
\end{array}\right) E_{2 n-4}+\left(\begin{array}{l}
n \\
6
\end{array}\right) E_{2 n-6}-\cdots .
$$

Let $S_{k}$ be the set of permutations $w=a_{1} a_{2} \cdots a_{2 n} \in \mathfrak{S}_{2 n}$ satisfying

$$
a_{1}>a_{2}<a_{3}>a_{4}<\cdots>a_{2 n-2 k}, \quad a_{2 n-2 k+1}>a_{2 n-2 k+2}>\cdots>a_{2 n},
$$

and let $T_{k}$ be those permutations in $S_{k}$ that also satisfy $a_{2 n-2 k}>a_{2 n-2 k+1}$. Hence $S_{1}-T_{1}$ consists of all alternating permutations in $\mathfrak{S}_{n}$. Moreover, $T_{i}=S_{i+1}-T_{i+1}$. Hence

$$
E_{n}=\#\left(S_{1}-T_{1}\right)=\# S_{1}-\#\left(S_{2}-T_{2}\right)=\cdots=\# S_{1}-\# S_{2}+\# S_{3}-\cdots .
$$

A permutation in $S_{k}$ is obtained by choosing $a_{2 n-2 k+1}, a_{2 n-2 k+2}, \ldots, a_{2 n}$ in $\left(\begin{array}{c}2 n \\ 2 k\end{array}\right)$ ways and then $a_{1}, a_{2}, \ldots, a_{2 n-2 k}$ in $E_{2(n-k)}$ ways. Hence $\# S_{k}=\left(\begin{array}{c}2 n \\ 2 k\end{array}\right) E_{2(n-k)}$, and the proof follows.

Third proof. Our third proof gives a more general formula that makes it more obvious why $\sec x$ appears in Theorem 1.1. Our second proof can also be extended to yield equation (1.6) below. A slightly more complicated argument, omitted here, explains the term $\tan x$. For some generalizations, see [36] and [70, Exer. 3.80].

Let $k, n \geq 1$, and let $f_{k}(n)$ denote the number of permutations $a_{1} a_{2} \cdots a_{k n} \in$ $\mathfrak{S}_{k n}$ satisfying $a_{i}>a_{i+1}$ if and only if $k \mid i$. Set

$$
F_{k}(x)=\sum_{n \geq 0} f_{k}(n) \frac{x^{k n}}{(k n) !}
$$

We claim that

$$
F_{k}(x)=\frac{1}{\sum_{n \geq 0}(-1)^{n} \frac{x^{k n}}{(k n) !}} .
$$

To prove equation (1.6), note that the number of permutations $b_{1} b_{2} \cdots b_{k n} \in \mathfrak{S}_{k n}$ such that $b_{i}>b_{i+1}$ only if (but not necessarily if) $k \mid i$ is easily seen to be the multinomial coefficient $\left(\begin{array}{c}k n \\ k, k, \ldots, k\end{array}\right)=(k n) ! / k !^{n}$. A straightforward inclusion-exclusion argument then gives

$$
f_{k}(n)=\sum_{j=1}^{n} \sum_{\substack{i_{1}+\cdots+i_{j}=n \\
i_{r}>0}}(-1)^{n-j}\left(\begin{array}{c}
k n \\
i_{1} k, \ldots, i_{j} k
\end{array}\right) .
$$

Comparing with the expansion

$$
\frac{1}{\sum_{n \geq 0}(-1)^{n} \frac{x^{k n}}{(k n) !}}=\sum_{j \geq 0}\left(\sum_{n \geq 1}(-1)^{n-1} \frac{x^{k n}}{(k n) !}\right)^{j}
$$

completes the proof. 
The inclusion-exclusion formula (1.7) can be regarded as the expansion of a determinant, giving the determinantal formula

$$
f_{k}(n)=(k n) ! \operatorname{det}[1 /(k(j-i+1)) !]_{i, j=1}^{n},
$$

where we set $1 /(-m) !=0$ for $m>0$. (See [70, pp. 69-71].) The case $k=2$ gives a formula for $E_{2 n}$. Similarly there holds

$$
E_{2 n-1}=(2 n-1) ! \operatorname{det}\left[1 / c_{i j} !\right]_{i, j=1}^{n},
$$

where $c_{1, j}=2 j-1$ and $c_{i j}=2(j-i+1)$ for $2 \leq i \leq n$.

Because $f(z)=\sec z+\tan z$ is a well-behaved function of the complex variable $z$ (in particular, it is meromorphic, and all poles are simple), it is routine to derive a precise asymptotic estimate of $E_{n}$. The smallest pole of $f(z)$ is at $z=\pi / 2$, with residue -2 , and the next smallest pole is at $z=-3 \pi / 2$. Hence

$$
\frac{E_{n}}{n !}=\frac{4}{\pi}\left(\frac{2}{\pi}\right)^{n}+O\left(\left(\frac{2}{3 \pi}\right)^{n}\right) .
$$

In fact, the poles of $f(z)$ are precisely $z=(-1)^{n}(2 n+1) \pi / 2, n \geq 0$, all with residue -2 , leading to the convergent asymptotic series

$$
\frac{E_{n}}{n !}=2\left(\frac{2}{\pi}\right)^{n+1} \sum_{k \geq 0}(-1)^{k(n+1)} \frac{1}{(2 k+1)^{n+1}} .
$$

This formula for $n$ odd is equivalent to the well-known evaluation of $\zeta(n+1):=$ $\sum_{k \geq 1} k^{-(n+1)}$. For further information on asymptotic expansions see [23], where in particular Euler numbers are treated in Example IV.35.

\section{Refinements of Euler numbers}

For the purposes of this paper, a refinement of the Euler number $E_{n}$ is a sequence $a_{0}, a_{1}, \ldots$ of nonnegative integers summing to $E_{n}$. Often we encode the sequence by a polynomial $P(q)=\sum a_{i} q^{i}$, so $P(1)=E_{n}$. This polynomial is sometimes called a $q$-analogue of $E_{n}$, especially if it involves such " $q$-objects" as the finite field $\mathbb{F}_{q}$ or expressions such as $[n] !:=(1-q)\left(1-q^{2}\right) \cdots\left(1-q^{n}\right)$ or $\sum a_{n} x^{n} /[n]$ !. We briefly discuss two refinements of $E_{n}$.

The first refinement provides an elegant scheme for computing the Euler numbers. Let $E_{n, k}$ denote the number of alternating permutations of $[n+1]$ with first term $k+1$. For instance, $E_{n, n}=E_{n}$. It is easy to verify the recurrence

$$
E_{0,0}=1, \quad E_{n, 0}=0(n \geq 1), \quad E_{n+1, k+1}=E_{n+1, k}+E_{n, n-k}(n \geq k \geq 0) .
$$

Note that if we place the $E_{n, k}$ 's in the triangular array

$$
\begin{array}{cccccccccc} 
& \multicolumn{1}{c}{E_{00}} & & & & \\
& & & E_{10} & \rightarrow & E_{11} & & & \\
& E_{22} & \leftarrow & E_{21} & \leftarrow & E_{20} & & \\
E_{44} & E_{30} & \rightarrow & E_{31} & \rightarrow & E_{32} & \rightarrow & E_{33} & \\
E_{43} & \leftarrow & E_{42} & \leftarrow & E_{41} & \leftarrow & E_{40}
\end{array}
$$

and read the entries in the direction of the arrows from top-to-bottom (the so-called boustrophedon or ox-plowing order), then the first number read in each row is 0 , 
and each subsequent entry is the sum of the previous entry and the entry above in the previous row. The first seven rows of the array are as follows:

$$
\begin{aligned}
& 1 \\
& 0 \rightarrow 1 \\
& 1 \leftarrow 1 \leftarrow 0 \\
& 0 \quad \rightarrow \quad 1 \quad \rightarrow \quad 2 \quad \rightarrow \quad 2 \\
& 5 \leftarrow 5 \leftarrow 4 \leftarrow 2 \leftarrow 0
\end{aligned}
$$

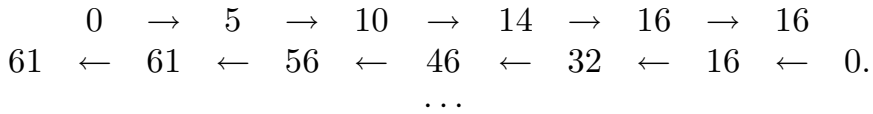

We can obtain a generating function for the number $E_{n, k}$ as follows. Define

$$
[m, n]=\left\{\begin{array}{cc}
m, & m+n \text { odd } \\
n, & m+n \text { even }
\end{array}\right.
$$

Then

$$
\sum_{m \geq 0} \sum_{n \geq 0} E_{m+n,[m, n]} \frac{x^{m}}{m !} \frac{y^{n}}{n !}=\frac{\cos x+\sin x}{\cos (x+y)} .
$$

For a proof see Graham, Knuth, and Patashnik [38, Exer. 6.75]. The numbers $E_{n, k}$ are called Entringer numbers, after R. C. Entringer [22]. The triangular array (2.1) is due to L. Seidel [63] (who used the word "boustrophedon" to describe the triangle). It was rediscovered by Kempner [44], Entringer [22] and Arnold [3]. For further information and references, see J. Millar, N. J. A. Sloane, and N. E. Young [51]. A more recent reference is R. Ehrenborg and S. Mahajan [20, §2].

Our second refinement $E_{n}(q)$ of Euler numbers is a natural $q$-analogue. An inversion of a permutation $w=a_{1} \cdots a_{n} \in \mathfrak{S}_{n}$ is a pair $(i, j)$ such that $i<j$ and $a_{i}>a_{j}$. Let $\operatorname{inv}(w)$ denote the number of inversions of $w$, and define

$$
E_{n}(q)=\sum_{w \in \operatorname{Ralt}_{n}} q^{\operatorname{inv}(w)}
$$

For instance, we have $\operatorname{inv}(1324)=1, \operatorname{inv}(1423)=2, \operatorname{inv}(2314)=2, \operatorname{inv}(2413)=3$, and $\operatorname{inv}(3412)=4$, so $E_{n}(q)=q+2 q^{2}+q^{3}+q^{4}$. Similarly define

$$
E_{n}^{*}(q)=\sum_{w \in \mathrm{Alt}_{n}} q^{\mathrm{inv}(w)}
$$

For instance, we have $\operatorname{inv}(2143)=2, \operatorname{inv}(3142)=3, \operatorname{inv}(3241)=4, \operatorname{inv}(4132)=4$, and $\operatorname{inv}(4231)=5$, so $E_{4}(q)=q^{2}+q^{3}+2 q^{4}+q^{5}$. Note that

$$
E_{n}^{*}(q)=q^{\left(\begin{array}{c}
n \\
2
\end{array}\right)} E_{n}(1 / q)
$$

an immediate consequence of the involution (1.1). The polynomials $E_{n}(q)$ for $2 \leq$ $n \leq 6$ are given by

$$
\begin{aligned}
& E_{2}(q)=1 \\
& E_{3}(q)=q+q^{2} \\
& E_{4}(q)=q^{2}+q^{3}+2 q^{4}+q^{5} \\
& E_{5}(q)=q^{2}+2 q^{3}+3 q^{4}+4 q^{5}+3 q^{6}+2 q^{7}+q^{8} \\
& E_{6}(q)=q^{3}+2 q^{4}+5 q^{5}+7 q^{6}+9 q^{7}+10 q^{8}+10 q^{9}+8 q^{10}+5 q^{11}+2 q^{12}+q^{13} .
\end{aligned}
$$


The main combinatorial result on the " $q$-Euler polynomials" $E_{n}(q)$ and $E_{n}^{*}(q)$, known to Schützenberger in 1975 (according to D. Foata) and rediscovered by Stanley [64] in the context of binomial posets, is the following. Define the $q$-cosine and $q$-sine functions by

$$
\begin{aligned}
\cos _{q}(x) & =\sum_{n \geq 0}(-1)^{n} \frac{x^{2 n}}{[2 n] !} \\
\sin _{q}(x) & =\sum_{n \geq 0}(-1)^{n} \frac{x^{2 n+1}}{[2 n+1] !}
\end{aligned}
$$

where $[m] !=(1-q)\left(1-q^{2}\right) \cdots\left(1-q^{m}\right)$. Note that these series become $\cos x$ and $\sin x$ after substituting $(1-q) x$ for $x$ and letting $q \rightarrow 1$. Similarly define the variants

$$
\begin{aligned}
\cos _{q}^{*}(x) & =\sum_{n \geq 0}(-1)^{n} q^{\left(\begin{array}{c}
2 n \\
2
\end{array}\right)} \frac{x^{2 n}}{[2 n] !} \\
\sin _{q}^{*}(x) & =\sum_{n \geq 0}(-1)^{n} q^{\left(\begin{array}{c}
2 n+1 \\
2
\end{array}\right)} \frac{x^{2 n+1}}{[2 n+1] !}
\end{aligned}
$$

Note that

$$
\cos _{q}^{*}(x)=\cos _{1 / q}(-x / q)
$$

and similarly for $\sin _{q}^{*}(x)$.

THEOREM 2.1. We have

$$
\begin{aligned}
& \sum_{n \geq 0} E_{n}(q) \frac{x^{n}}{[n] !}=\frac{1}{\cos _{q}(x)}+\frac{\sin _{q}(x)}{\cos _{q}(x)} \\
& \sum_{n \geq 0} E_{n}^{*}(q) \frac{x^{n}}{[n] !}=\frac{1}{\cos _{q}^{*}(x)}+\frac{\sin _{q}^{*}(x)}{\cos _{q}^{*}(x)}
\end{aligned}
$$

One way to prove Theorem 2.1 is by a straightforward generalization of the third proof of Theorem 1.1. A more conceptual explanation for this result and some generalizations based on binomial posets appear in [64]. Note that the result for $E_{n}^{*}(q)$ is an immediate consequence of that for $E_{n}(q)$ and equations $(2.4)$ and (2.5). The reasoning used to deduce the determinantal formulas (1.8) and (1.9) from equation (1.7) can also be straightforwardly generalized to give

$$
\begin{aligned}
E_{2 n}(q) & =[2 n-1] ! \operatorname{det}[1 /[2(j-i+1)] !]_{i, j=1}^{n} \\
E_{2 n-1}(q) & =[2 n-1] ! \operatorname{det}\left[1 /\left[c_{i j}\right] !\right]_{i, j=1}^{n},
\end{aligned}
$$

where $c_{1, j}=2 j-1$ and $c_{i j}=2(j-i+1)$ for $2 \leq i \leq n$.

The polynomials $E_{n}(q)$ also appear in a natural way in the theory of symmetric functions. Assuming familiarlity with this theory $[\mathbf{4 9}][\mathbf{7 2}, \mathrm{Ch} .7]$, let $\tau_{n}$ be as in Section 3.6 of this paper, and let $s_{\tau_{n}}$ be the corresponding skew Schur function. Then

$$
E_{n}(q)=[n] ! s_{\tau_{n}}\left(1, q, q^{2}, \ldots\right) .
$$

It follows from standard properties of Schur functions (see [72, Prop. 7.19.11]) that $E_{n}(q)$ has the additional combinatorial interpretation

$$
E_{n}(q)=\sum_{w \in \operatorname{Alt}_{n}} q^{\operatorname{maj}\left(w^{-1}\right)},
$$



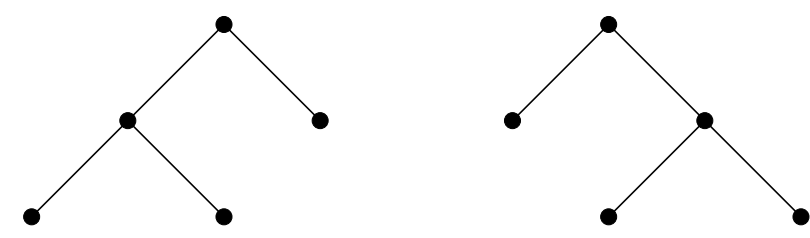

Figure 1. The two complete binary trees with five vertices

where

$$
\operatorname{maj}(w)=\sum_{\substack{1 \leq i \leq n-1 \\ a_{i}>a_{i+1}}} i,
$$

the major index of $w=a_{1} \cdots a_{n}$. The equivalence of equations (2.3) and (2.8) is also a consequence of well-known properties of inv and maj (e.g., [71, Thm. 1.4.8]). In the context of symmetric functions, the determinantal formulas (2.6) and (2.7) are consequences of the Jacobi-Trudi identity $[\mathbf{7 2}, \S 7.16]$ for the skew Schur function $s_{\tau_{n}}$.

A number of other $q$-analogues of $E_{n}$ (sometimes just for $n$ odd) have been proposed; see $[\mathbf{2 5}][\mathbf{3 1}][\mathbf{4 0}][\mathbf{4 3}][\mathbf{5 8}][\mathbf{5 9}]$ for further information.

\section{Other occurrences of Euler numbers}

There are numerous occurrences of Euler numbers not directly related to alternating permuations. We discuss a few of these here. For more information on this topic, see the treatise $[\mathbf{8 2}]$ of Viennot.

3.1. Complete increasing binary trees. A (plane) binary tree on the vertex set $[n]$ is defined recursively by having a root vertex $v$ and a left and right subtree of $v$ which are themselves binary trees or are empty. A binary tree is complete if every vertex either has two children or is an endpoint. A binary tree on the vertex set $[n]$ is increasing if every path from the root is increasing. Figure 1 shows the two complete binary trees with five vertices. Each one has eight increasing labelings, so there are 16 complete increasing binary trees on the vertex set [5].

THEOREM 3.1. The number of complete increasing binary trees on $[2 m+1]$ is the Euler number $E_{2 m+1}$. (There is a similar but more complicated statement for the vertex set $[2 m]$ which we do not give here.)

Proof. Given a sequence $u=a_{1} a_{2} \cdots a_{n}$ of distinct integers, define a labelled binary tree $T_{u}$ as follows. Let $a_{i}=\min \left\{a_{1}, \ldots, a_{n}\right\}$. Let $a_{i}$ be the root of $T_{w}$, and recursively define the left subtree of the root to be $T_{a_{1} \cdots a_{i-1}}$, and the right subtree of the root to be $T_{a_{i+1} \cdots a_{n}}$. It is not hard to check that the map $w \mapsto T_{w}$ is a bijection from alternating permutations $w \in \mathfrak{S}_{2 m+1}$ to complete increasing binary trees on $[2 m+1]$.

3.2. Flip equivalence. The Euler numbers are related to increasing binary trees in another way. The flip of a binary tree at a vertex $v$ is the binary tree obtained by interchanging the left and right subtrees of $v$. Define two increasing binary trees $T$ and $T^{\prime}$ on the vertex set $[n]$ to be flip equivalent if $T^{\prime}$ can be obtained from $T$ by a sequence of flips. Clearly this definition of flip equivalence is an equivalence relation. The equivalence classes are in an obvious bijection with 

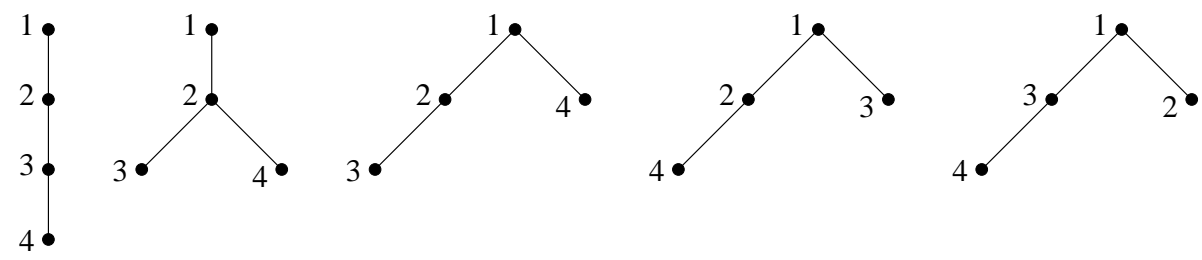

Figure 2. The five increasing 1-2 trees with four vertices

increasing 1-2 trees on the vertex set $[n]$, that is, increasing (rooted) trees so that every non-endpoint vertex has one or two children. (These are not plane trees, i.e., the order in which we write the children of a vertex is irrelevant.) Figure 2 shows the five increasing 1-2 trees on four vertices, so $f(4)=5$.

Theorem 3.2. We have $f(n)=E_{n}$ (an Euler number).

Proof. Perhaps the most straightforward proof is by generating functions. (A combinatorial proof was first given by Donaghey [18].) Let

$$
y=\sum_{n \geq 1} f(n) \frac{x^{n}}{n !}=x+\frac{x^{2}}{2}+2 \frac{x^{3}}{6}+\cdots .
$$

Then $y^{\prime}=\sum_{n>0} f(n+1) x^{n} / n$ !. Every increasing 1-2 tree with $n+1$ vertices is either (a) a single vertex $(n=0)$, (b) has one subtree of the root which is an increasing binary tree with $n$ vertices, or (c) has two subtrees of the root, each of which is an increasing binary tree, with $n$ vertices in all. The order of the two subtrees is irrelevant. From this observation we obtain the differential equation $y^{\prime}=1+y+\frac{1}{2} y^{2}, y(0)=0$. The unique solution is $y=\sec x+\tan x-1$, and the proof follows from Theorem 1.1.

Much additional information concerning the connection between alternating permutations and increasing trees appears in a paper of Kuznetsov, Pak, and Postnikov [46].

AlgEBRAIC NOTE. Let $\mathcal{T}_{n}$ be the set of all increasing binary trees with vertex set $[n]$. For $T \in \mathcal{T}_{n}$ and $1 \leq i \leq n$, let $\omega_{i} T$ be the flip of $T$ at vertex $i$. Then clearly the $\omega_{i}$ 's generate a group isomorphic to $(\mathbb{Z} / 2 \mathbb{Z})^{n}$ acting on $\mathcal{T}_{n}$, and the orbits of this action are the flip equivalence classes. For further details see Foata [24] and Foata-Strehl [27].

3.3. Ballot sequences. A ballot sequence of length $2 n$ is a sequence $b_{1}, b_{2}, \ldots$, $b_{2 n}$ of $n 1$ 's and $n-1$ 's for which all partial sums are nonnegative. The number of ballot sequences of length $2 n$ is the Catalan number $C_{n}$ [72, Cor. 6.2.3(ii)]. Given a ballot sequence $b=b_{1}, b_{2}, \ldots, b_{2 n}$, define

$$
\begin{aligned}
\omega(b) & =\prod_{i: b_{i}=1}\left(b_{1}+b_{2}+\cdots+b_{i}\right) \\
\omega^{*}(b) & =\prod_{i: b_{i}=1}\left(b_{1}+b_{2}+\cdots+b_{i}+1\right) .
\end{aligned}
$$

For instance, if $b=(1,1,-1,1,-1,-1,1,-1)$ then $\omega(b)=1 \cdot 2 \cdot 2 \cdot 1=4$ and $\omega^{*}(b)=2 \cdot 3 \cdot 3 \cdot 2=36$. Let $B(n)$ denote the set of all ballot sequences of length 
$2 n$. It follows from a bijection of Françon and Viennot [30] between binary trees and weighted paths (also explained in $[\mathbf{3 7}, \S 5.2]$ ) that

$$
\begin{aligned}
\sum_{b \in B(n)} \omega(b)^{2} & =E_{2 n} . \\
\sum_{b \in B(n)} \omega(b) \omega^{*}(b) & =E_{2 n+1} .
\end{aligned}
$$

Equation (3.1) suggests the following question: is there a finite-dimensional algebra $\mathcal{A}_{n}$ (say over $\mathbb{C}$ ) with a "natural" combinatorial definition whose irreducible representations have dimension $\omega(b), b \in B(n)$ ? It would then follow that $\operatorname{dim} \mathcal{A}_{n} / \sqrt{\mathcal{A}_{n}}=E_{2 n}$, where $\sqrt{\mathcal{A}_{n}}$ denotes the radical of $\mathcal{A}_{n}$.

3.4. Simsun permutations. Define a simsun permutation to be a permutation $w=a_{1} \cdots a_{n} \in \mathfrak{S}_{n}$ such that for all $1 \leq k \leq n$, the subword of $w$ consisting of $1,2, \ldots, k$ (in the order they appear in $w$ ) does not have three consecutive decreasing elements. For instance, $w=425163$ is not simsun since if we remove 5 and 6 from $w$ we obtain 4213 , which contains the three consecutive decreasing elements 421. Simsun permutations were named after Rodica Simion and Sheila Sundaram and were first described in print by Sundaram [77, §3]. They are a variant of a class of permutations due to Foata and Schützenberger [26] known as André permutations. We have chosen here to deal only with simsun permutations because their definition is a little simpler than that of André permutations. Simion and Sundaram prove in their paper the following basic result on simsum permutations.

THEOREM 3.3. The number of simsun permutations in $\mathfrak{S}_{n}$ is the Euler number $E_{n+1}$.

PROOF (sketch). Let $f_{k}(n)$ be the number of simsun permutations $w=a_{1} \cdots a_{n} \in$ $\mathfrak{S}_{n}$ with $k$ descents, i.e., $k$ values of $i$ for which $a_{i}>a_{i+1}$. By inserting $n+1$ into a simsun permutation in $\mathfrak{S}_{n}$, we get the recurrence

$$
f_{k}(n+1)=(n-2 k+2) f_{k-1}(n)+(k+1) f_{k}(n),
$$

with the initial conditions $f_{0}(1)=1, f_{k}(n)=0$ for $k>\lfloor n / 2\rfloor$. The proof now follows from routine generating function arguments.

Another proof of Theorem 3.3 was given by Maria Monks (private communication, 2008). She gives a bijection between simsun permutations in $\mathfrak{S}_{n}$ and the oriented increasing binary trees on the vertex set $[n]$ discussed in Section 3.2. Simsun permutations have an interesting connection with the $c d$-index of $\mathfrak{S}_{n}$, discussed in Section 6. For some further work on simsun permutations, see Chow and Shiu [14] and Deutsch and Elizalde [16].

3.5. Orbits of chains of partitions. A partition $\pi$ of the set $[n]$ is a collection $\left\{B_{1}, \ldots, B_{k}\right\}$ of nonempty subsets of $[n]$ (called the blocks of $\pi$ ) such that $\bigcup B_{i}=[n]$ and $B_{i} \cap B_{j}=\emptyset$ if $i \neq j$. Let $\Pi_{n}$ denote the set of all partitions of [n]. If $\pi, \sigma \in \Pi_{n}$, then we say that $\pi$ is a refinement of $\sigma$, denoted $\pi \leq \sigma$, if every block of $\pi$ is contained in a block of $\sigma$. The relation $\leq$ is a partial order, so $\Pi_{n}$ becomes a partially ordered set (poset). Note that the symmetric group $\mathfrak{S}_{n}$ acts on $\Pi_{n}$ in an obvious way, viz., if $B=\left\{a_{1}, \ldots, a_{j}\right\}$ is a block of $\pi$ and $w \in \mathfrak{S}_{n}$, then $w \cdot B:=\left\{w\left(a_{1}\right), \ldots, w\left(a_{j}\right)\right\}$ is a block of $w \cdot \pi$. 

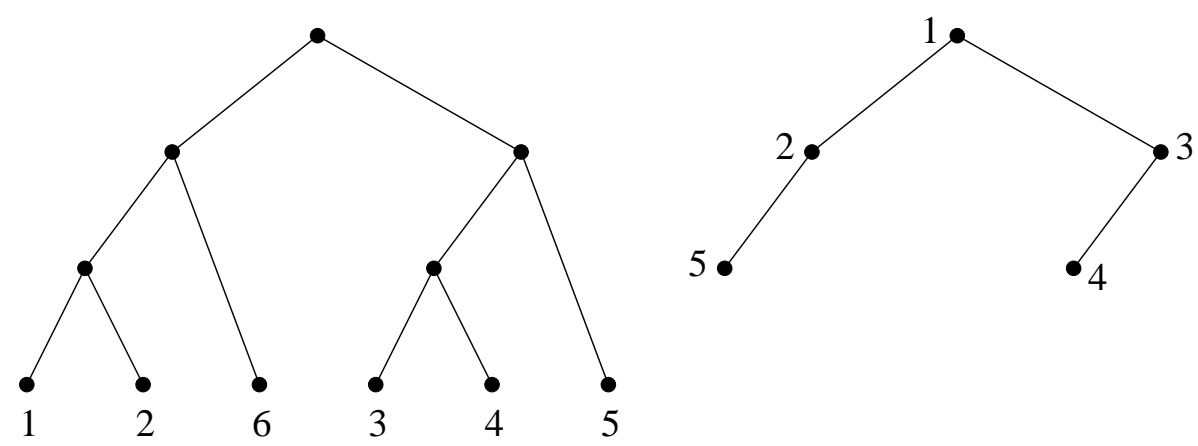

Figure 3. Two trees associated with a maximal chain in $\Pi_{6}$

Let $\mathcal{M}\left(\Pi_{n}\right)$ denote the set of all maximal chains of $\Pi_{n}$, i.e., all chains

$$
\pi_{0}<\pi_{1}<\cdots<\pi_{n-1}
$$

so that for all $0 \leq i \leq n-2, \pi_{i+1}$ is obtained from $\pi_{i}$ by merging two blocks of $\pi_{i}$. Thus $\pi_{i}$ has exactly $n-i$ blocks. In particular, $\pi_{0}$ is the partition into $n$ singleton blocks, and $\pi_{n-1}$ is the partition into one block $[n]$. The action of $\mathfrak{S}_{n}$ on $\Pi_{n}$ induces an action on $\mathcal{M}\left(\Pi_{n}\right)$. For instance, when $n=5$ a class of orbit representatives is given by the five chains below. We write e.g. $12-34-5$ for the partition $\{\{1,2\},\{3,4\},\{5\}\}$, and we omit the first and last element of each chain.

$$
\begin{aligned}
& 12-3-4-5<123-4-5<1234-5 \\
& 12-3-4-5<123-4-5<123-45 \\
& 12-3-4-5<12-34-5<125-34 \\
& 12-3-4-5<12-34-5<12-345 \\
& 12-3-4-5<12-34-5<1234-5
\end{aligned}
$$

THEOREM 3.4. The number of orbits of the action of $\mathfrak{S}_{n}$ on $\mathcal{M}\left(\Pi_{n}\right)$ is the Euler number $E_{n-1}$.

Proof. Given a maximal chain $\mathfrak{m}$ in $\mathcal{M}\left(\Pi_{n}\right)$, define a binary tree with endpoints $1,2, \ldots, n$ by the rule that for each internal vertex $v$, at some point in the chain we merged together a block consisting of the endpoints of the left subtree of $v$ with a block consisting of the endpoints of the right subtree of $v$. For instance, if the maximal chain is (omitting the first and last elements) $12-3-4-5-6,12-34-5-6$, $12-345-6,126-345$, then the tree is given by Figure 3(a). Label each internal vertex $v$ by $n-i$ if that vertex was created at the $i$ th step of the merging process and delete the endpoints, as illustrated in Figure 3(b), resulting in an increasing binary tree $T_{\mathfrak{m}}$ on vertices $1,2, \ldots, n$. The tree $T_{\mathfrak{m}}$ is well-defined up to flip equivalence. Two maximal chains $\mathfrak{m}$ and $\mathfrak{m}^{\prime}$ belong to the same $\mathfrak{S}_{n}$-orbit if and only $T_{\mathfrak{m}}$ and $T_{\mathfrak{m}^{\prime}}$ are flip equivalent, and the proof follows from Theorem 3.2.

Theorem 3.4 was first proved by Stanley [67, Thm. 7.7] by showing that the number of orbits satisfied the recurrence (1.4). By elementary representation theory, the number of orbits of $\mathfrak{S}_{n}$ acting on $\mathcal{M}\left(\Pi_{n}\right)$ is the multiplicity of the trivial representation in this action. This observation suggests the problem of decomposing $\mathfrak{S}_{n}$-actions on various sets of chains in $\Pi_{n}$ into irreducible representations. The first 


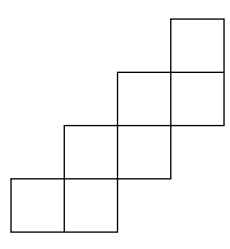

$\tau_{7}$

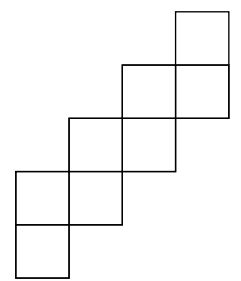

$\tau_{8}$

Figure 4 . The zigzag shapes $\tau_{7}$ and $\tau_{8}$

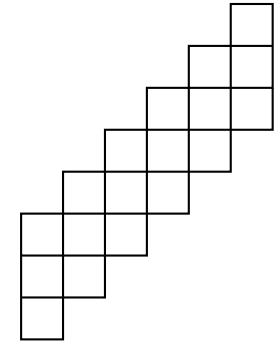

$\sigma_{18}$

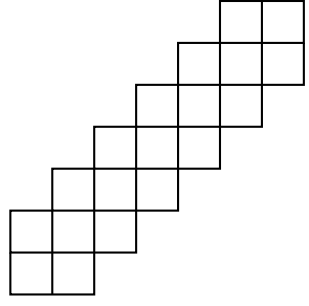

$\sigma_{19}$

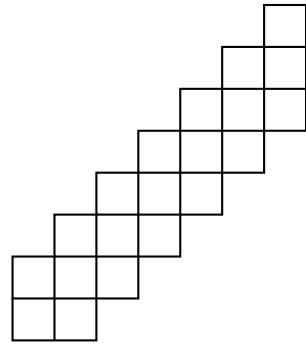

$\sigma_{20}$

Figure 5 . The thickened zigzag shapes $\sigma_{18}, \sigma_{19}$, and $\sigma_{20}$

results in this direction appear in $[\mathbf{6 7}, \S 7]$. Many further results were obtained by Sundaram [77]. Another such result is Theorem 3.7 below.

3.6. Thickened zigzag tableaux. For this subsection we assume familiarity with the theory of symmetric functions such as developed in [49][72, Chap. 7]. Let $\tau_{n}$ be the border strip (or ribbon) corresponding to the composition $\alpha=$ $(1,2,2, \ldots, 2, j)$ of $n$, where $j=1$ if $n$ is even and $j=2$ if $n$ is odd. Thus the (Young) diagram of $\tau_{n}$ has a total of $n$ squares. Figure 4 shows the diagrams of $\tau_{7}$ and $\tau_{8}$. We call $\tau_{n}$ a zigzag shape.

Given any skew shape $\lambda / \mu$ of size $n$, let $f^{\lambda / \mu}$ denote the number of standard Young tableaux (SYT) of shape $\lambda / \mu$, i.e., the number of ways to put $1,2, \cdots, n$ into the squares of (the diagram of) $\lambda / \mu$, each number $1,2, \ldots, n$ occuring exactly once, so that the rows and colums are increasing. If $T$ is an SYT of shape $\tau_{n}$, then reading the numbers of $T$ from top-to-bottom and right-to-left gives a bijection with reverse alternating permutations in $\mathfrak{S}_{n}$. Hence

$$
f^{\tau_{n}}=E_{n} .
$$

Y. Baryshnikov and D. Romik [7] give a surprising generalization of equation (3.2) in which the shapes $\tau_{n}$ are "thickened." We only mention the simplest case here. The relevant shapes $\sigma_{n}$ (with $n$ squares) are illustrated in Figure 5, for each of the three case $n \equiv 0,1,2(\bmod 3)$. 
THEOREM 3.5. We have

$$
\begin{aligned}
f^{\sigma_{3 n-2}} & =\frac{(3 n-2) ! E_{2 n-1}}{(2 n-1) ! 2^{2 n-2}} \\
f^{\sigma_{3 n-1}} & =\frac{(3 n-1) ! E_{2 n-1}}{(2 n-1) ! 2^{2 n-1}} \\
f^{\sigma_{3 n}} & =\frac{(3 n) !\left(2^{2 n-1}-1\right) E_{2 n-1}}{(2 n-1) ! 2^{2 n-1}\left(2^{2 n}-1\right)} .
\end{aligned}
$$

The proof of Theorem 3.5 generalizes a transfer operator approach to alternating permutations due to Elkies [21]. Is there a bijective proof?

3.7. Möbius functions. Let $P$ be a finite poset and $\operatorname{Int}(P)$ the set of nonempty closed invervals $[s, t]=\{u: s \leq u \leq t\}$ of $P$. The Möbius function of $P$ (say over $\mathbb{R})$ is the function $\mu: \operatorname{Int}(P) \rightarrow \mathbb{R}$ defined recursively as follows:

$$
\begin{aligned}
\mu(t, t) & =1, \quad \text { for all } t \in P \\
\sum_{u \in[s, t]} \mu(s, u) & =0, \text { for all } s<t \text { in } P .
\end{aligned}
$$

Here we write $\mu(s, u)$ for $\mu([s, u])$. The Möbius function has many important properties and applications. See for instance [70, Chap. 3] for more information. A number of posets have Euler numbers as Möbius function values (up to sign). We state the two most significant such results here. Both these results are special cases of much more general results that are related to topological combinatorics and representation theory.

For $n \geq 1$ let $B_{n, 2}$ denote the poset of all subsets of $[n]$ with an even number of elements, ordered by inclusion, with a unique maximal element $\hat{1}$ adjoined when $n$ is odd. Thus $B_{n, 2}$ has a unique minimal element $\emptyset$ and a unique maximal element, which we denote by $\hat{1}$ even if $n$ is even. Figure 6 shows $B_{5,2}$.

TheOREM 3.6. For the poset $B_{n, 2}$ we have

$$
\mu(\emptyset, \hat{1})=(-1)^{\lceil n / 2\rceil} E_{n} .
$$

Theorem 3.6 is best understood in the general context of rank-selected subposets of a graded poset. For an introduction to this theory see [70, §3.12].

Now let $\Pi_{2 n, 2}$ denote the subposet of the poset $\Pi_{2 n}$ defined in Section 3.5 consisting of those partitions whose block sizes are all even, with a minimal element $\hat{0}$ adjoined. This poset has a unique maximal element $\hat{1}$ consisting of the partition with one block $[2 n]$.

THEOREM 3.7. For the poset $\Pi_{2 n, 2}$ we have

$$
\mu(\hat{0}, \hat{1})=(-1)^{n} E_{2 n-1} .
$$

Theorem 3.7 is due to G. Sylvester [78]. For some generalizations see [12] and [65].

3.8. Polytope volumes. Euler numbers occur as (normalized) volumes of certain convex polytopes. The first polytope, which we call the zigzag order polytope $\mathcal{P}_{n}$, consists of all points $x=\left(x_{1}, \ldots, x_{n}\right) \in \mathbb{R}^{n}$ satisfying

$$
\begin{aligned}
& 0 \leq x_{i} \leq 1, \quad 1 \leq i \leq n \\
& x_{1} \geq x_{2} \leq x_{3} \geq \cdots x_{n} .
\end{aligned}
$$




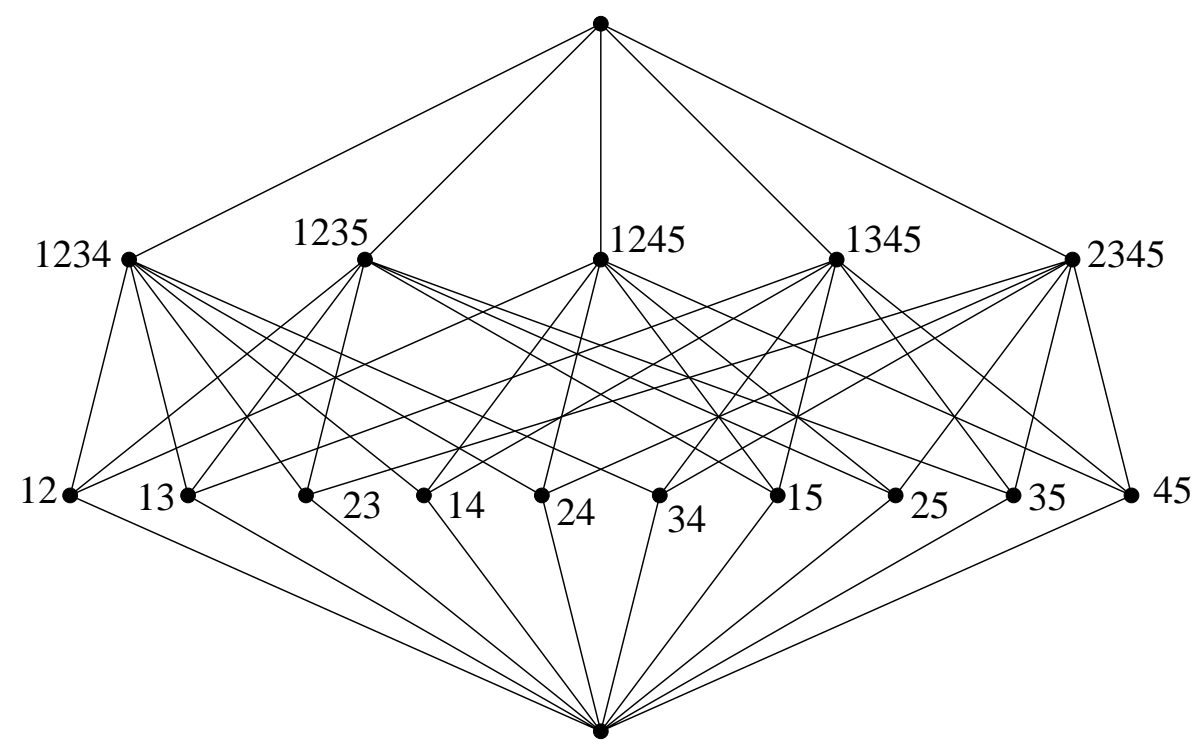

FIgURE 6 . The poset $B_{5,2}$

To compute its volume, for each alternating permutation $w=a_{1} a_{2} \cdots a_{n} \in \mathfrak{S}_{n}$, let $w^{-1}=b_{1} b_{2} \cdots b_{n}$. Let

$$
\mathcal{P}_{w}=\left\{\left(x_{1}, \ldots, x_{n}\right) \in \mathcal{P}_{n}: x_{b_{1}} \leq x_{b_{2}} \leq \cdots \leq x_{b_{n}}\right\} .
$$

It is easy to see that each $\mathcal{P}_{w}$ is a simplex with volume $1 / n$ !. One can check using the theory of $P$-partitions $[\mathbf{7 0}, \S 4.5]$ that the $\mathcal{P}_{w}$ 's have disjoint interiors and union $\mathcal{P}_{n}$. Hence $\operatorname{vol}\left(\mathcal{P}_{n}\right)=E_{n} / n$ !.

The second polytope is called the zigzag chain polytope $\mathcal{C}_{n}$. It consists of all points $x=\left(x_{1}, \ldots, x_{n}\right) \in \mathbb{R}^{n}$ satisfying

$$
\begin{gathered}
x_{i} \geq 0, \quad 1 \leq i \leq n \\
x_{i}+x_{i+1} \leq 1, \quad 1 \leq i \leq n-1 .
\end{gathered}
$$

The polytope $\mathcal{C}_{n}$ first arose in [66] and [17]. It is also a special case of the Fibonacci polytopes defined by Rispoli [60]. A "naive" method for computing the volume is the following. For $0 \leq t \leq 1$ let

$$
f_{n}(t)=\int_{x_{1}=0}^{t} \int_{x_{2}=0}^{1-x_{1}} \int_{x_{3}=0}^{1-x_{2}} \cdots \int_{x_{n}=0}^{1-x_{n-1}} d x_{1} d x_{2} \cdots d x_{n} .
$$

Clearly $f(1)=\operatorname{vol}\left(\mathcal{C}_{n}\right)$. Differentiating equation (3.3) yields $f_{n}^{\prime}(t)=f_{n-1}(1-t)$. There are various ways to solve this recurrence for $f_{n}(t)$ (with the initial conditions $f_{0}(t)=1$ and $f_{n}(0)=0$ for $\left.n>0\right)$, yielding

$$
\sum_{n \geq 0} f_{n}(t) x^{n}=(\sec x)(\cos (t-1) x+\sin t x) .
$$

Putting $t=1$ gives

$$
\sum_{n \geq 0} f_{n}(1) x^{n}=\sec x+\tan x,
$$

so we conclude that $\operatorname{vol}\left(\mathcal{C}_{n}\right)=E_{n} / n$ ! 
A more sophisticated proof uses the earlier obtained fact that $\operatorname{vol}\left(\mathcal{P}_{n}\right)=E_{n} / n$ !. Given $\left(x_{1}, \ldots, x_{n}\right) \in \mathbb{R}_{n}$, define $\varphi\left(x_{1}, \ldots, x_{n}\right)=\left(y_{1}, \ldots, y_{n}\right) \in \mathbb{R}^{n}$ by

$$
y_{i}=\left\{\begin{aligned}
1-x_{i}, & \text { if } i \text { is odd } \\
x_{i}, & \text { if } i \text { is even. }
\end{aligned}\right.
$$

It is easily checked that $\varphi$ is an affine transformation taking $\mathcal{P}_{n}$ onto $\mathcal{C}_{n}$. Since the homogeneous part of $\varphi$ has determinant \pm 1 , it follows that $\varphi$ is a volume-preserving bijection from $\mathcal{P}_{n}$ onto $\mathcal{C}_{n}, \operatorname{so} \operatorname{vol}\left(\mathcal{C}_{n}\right)=\operatorname{vol}\left(\mathcal{P}_{n}\right)=E_{n} / n$ !. This argument appeared in Stanley [68, Thm. 2.3 and Exam. 4.3]. Chebikin and Ehrenborg[13] compute the $f$-vector (which gives the number of faces of each dimension) of a generalization of the polytopes $\mathcal{P}_{n}$. Since $\mathcal{P}_{n}$ and $\mathcal{C}_{n}$ are affinely equivalent, this computation also gives the $f$-vector of $\mathcal{C}_{n}$.

The polytope $\mathcal{C}_{n}$ has an interesting connection to tridiagonal matrices. An $n \times n$ matrix $M=\left(m_{i j}\right)$ is tridiagonal if $m_{i j}=0$ whenever $|i-j| \geq 2$. Let $\mathcal{T}_{n}$ be the set of all $n \times n$ tridiagonal doubly stochastic matrices $M$, i.e., $n \times n$ (real) tridiagonal matrices with nonnegative entries and with row and column sums equal to 1 . Thus $\mathcal{T}_{n}$ is a convex polytope in a real vector space of dimension $n^{2}$ (or of dimension $3 n-2$ if we discard coordinates that are always 0 ). It is easy to see that if we choose the $n-1$ entries $m_{12}, m_{23}, \ldots, m_{n-1, n}$ arbitrarily, then they determine a unique tridiagonal matrix $M$ with row and column sums 1 . Moreover, in order for $M$ to be doubly stochastic it is necessary and sufficient that $m_{i, i+1} \geq 0$ and

$$
m_{12}+m_{23} \leq 1, \quad m_{23}+m_{34} \leq 1, \ldots, m_{n-2, n-1}+m_{n-1, n} \leq 1 .
$$

It follows that $\mathcal{T}_{n}$ is linearly equivalent to $\mathcal{C}_{n-1}$ (in fact, $\mathcal{T}_{n}$ projects bijectively to $\mathcal{C}_{n-1}$ ). Moreover, the relative volume of $\mathcal{T}_{n}$ (volume normalized so that a fundamental parallelopiped of the lattice $\operatorname{aff}\left(\mathcal{T}_{n} \cap \mathbb{Z}^{n^{2}}\right.$ has volume 1 , where aff denotes affine span) is $E_{n-1} /(n-1)$ !.

The $n \times n$ tridiagonal doubly-stochastic matrices form a face of the Birkhoff polytope $\mathcal{B}_{n}$ of all $n \times n$ doubly stochastic matrices. Another face of $\mathcal{B}_{n}$ with an interesting volume is the Chan-Robbins polytope $\mathcal{C R}_{n}$ defined by

$$
\mathcal{C R}_{n}=\left\{M=\left(m_{i j}\right) \in \mathcal{B}_{n}: m_{i j}=0 \text { if } i-j \geq 2\right\},
$$

for which $\operatorname{vol}\left(\mathcal{C R}_{n}\right)=C_{1} C_{2} \cdots C_{n} /\left(\begin{array}{l}n \\ 2\end{array}\right)$ ! (where $C_{i}$ is a Catalan number) $[\mathbf{6}][\mathbf{8 5}]$. The nice formulas for the volumes of $\mathcal{T}_{n}$ and $\mathcal{C R}_{n}$ suggest the problem of finding an interpolation between the two. For instance, for $1 \leq k \leq n-1$, can one compute the volume of the polytope

$$
\mathcal{T}_{n, k}=\left\{M=\left(m_{i j}\right) \in \mathcal{B}_{n}: m_{i j}=0 \text { if } i-j \geq 2 \text { or } j-i>k\right\} ?
$$

Note that $\mathcal{T}_{n, n-1}=\mathcal{B}_{n}$ and $\mathcal{T}_{n, 1}=\mathcal{C R}_{n}$.

3.9. Singularities. V. I. Arnold [3] (see also [4] for a followup) has shown that the Euler number $E_{n+1}$ is equal to the number of components of the space of real polynomials $f(x)=x^{n}+a_{1} x^{n-1}+\cdots+a_{n-1} x$ whose critical points (zeros of $\left.f^{\prime}(x)\right)$ are all real and whose $n-1$ critical values (the numbers $f(c)$ where $c$ is a critical point) are all different. For instance, when $n=3$ the polynomials $x^{3}+a x^{2}+b x$ form a real plane. The critical points are real if and only if $b \leq a^{2} / 3$. Two critical values coincide in the region $b<a^{2} / 3$ if and only if $b=a^{2} / 4$ or $b=0$. These two curves cut the region $b<a^{2} / 3$ into $E_{4}=5$ components. Arnold interprets this result in terms of morsifications of the function $x^{n+1}$; see his paper for further details. Arnold goes on to deduce a number of interesting properties 
of Euler numbers. He also extends the theory to morsifications of the functions $x^{n}+y^{2}$ and $x y+y^{n}$, thereby producing $B_{n}$ and $C_{n}$ analogues of Euler numbers (which correspond to the root system $A_{n}$ ).

\section{Longest alternating subsequences}

Much work has been devoted to the properties of the length is $(w)$ of the longest increasing subsequence of a permutation $a_{1} \cdots a_{n}$, i.e., the largest $k$ for which there exist $i_{1}<\cdots<i_{k}$ and $a_{i_{1}}<\cdots<a_{i_{k}}$. For a survey of this subject, see [73]. Two of the highlights of this subject are the following. Let $E(n)$ denote the expected length of the longest increasing subsequence of $w \in \mathfrak{S}_{n}$ (with respect to the uniform distribution on $\mathfrak{S}_{n}$ ). In symbols,

$$
E(n)=\frac{1}{n !} \sum_{w \in \mathfrak{S}_{n}} \text { is }(w) .
$$

It was shown by Vershik and Kerov [80], and the difficult part of the argument independently by Logan and Shepp $[\mathbf{4 8}]$, that

$$
E(n) \sim 2 \sqrt{n} .
$$

The notation $f(n) \sim g(n)$ means that $\lim _{n \rightarrow \infty} f(n) / g(n)=1$.

A far-reaching improvement of equation (4.1) was given by Baik, Deift, and Johansson [5], namely, they determined the (suitably scaled) limiting distribution of is $(w)$ for $w \in \mathfrak{S}_{n}$ as $n \rightarrow \infty$. Let $F(t)$ denote the Tracy-Widom distribution, a probability distribution on $\mathbb{R}$ first arising in the work of Tracy and Widom on eigenvalues of random hermitian matrices [79]. (We will not define this distribution here.) Write $\operatorname{is}_{n}(w)$ for is $(w)$, where $w \in \mathfrak{S}_{n}$. The result of Baik, Deift, and Johansson asserts that

$$
\lim _{n \rightarrow \infty} \operatorname{Prob}\left(\frac{\operatorname{is}_{n}(w)-2 \sqrt{n}}{n^{1 / 6}} \leq t\right)=F(t) .
$$

Here for each $n$ we are choosing $w \in \mathfrak{S}_{n}$ independently and uniformly.

We can ask whether similar results hold for alternating subsequences of $w \in \mathfrak{S}_{n}$. In particular, for $w \in \mathfrak{S}_{n}$ define as $(w)$ (or $\operatorname{as}_{n}(w)$ to make it explicit that $w \in \mathfrak{S}_{n}$ ) to be the length of the longest alternating subsequence of $w$. For instance, if $w=56218347$ then $\operatorname{as}(w)=5$, one alternating subsequence of longest length being 52834. Our source for material in this section is the paper [75].

It turns out that the behavior of as $(w)$ is much simpler than that of is $(w)$. The primary reason for this is the following lemma, whose straightforward proof we omit.

Lemma 4.1. Let $w \in \mathfrak{S}_{n}$. Then there is an alternating subsequence of $w$ of maximum length that contains $n$.

Lemma 4.1 allows us to obtain explicit formulas by induction. More specifically, define

$$
\begin{aligned}
a_{k}(n) & =\#\left\{w \in \mathfrak{S}_{n}: \operatorname{as}(w)=k\right\} \\
b_{k}(n) & =a_{1}(n)+a_{2}(n)+\cdots+a_{k}(n) \\
& =\#\left\{w \in \mathfrak{S}_{n}: \operatorname{as}(w) \leq k\right\} .
\end{aligned}
$$


For instance, $b_{1}(n)=1$, corresponding to the permutation $1,2, \ldots, n$, while $b_{2}(n)=$ $2^{n-1}$, corresponding to the permutations $u_{1}, u_{2} \ldots, u_{i}, n, v_{1}, v_{2}, \ldots, v_{n-i-1}$, where $u_{1}<u_{2}<\cdots<u_{i}$ and $v_{1}>v_{2}>\cdots>v_{n-i-1}$. Using Lemma 4.1, we can obtain the following recurrence for the numbers $a_{k}(n)$, together with the intial condition $a_{0}(0)=1$ :

$$
a_{k}(n+1)=\sum_{j=0}^{n}\left(\begin{array}{l}
n \\
j
\end{array}\right) \sum_{\substack{2 r+s=k-1 \\
r, s \geq 0}}\left(a_{2 r}(j)+a_{2 r+1}(j)\right) a_{s}(n-j) .
$$

This recurrence can be used to obtain the following generating function for the numbers $a_{k}(n)$ and $b_{k}(n)$. No analogous formula is known for increasing subsequences.

THEOREM 4.2. Let

$$
\begin{aligned}
A(x, t) & =\sum_{k, n \geq 0} a_{k}(n) t^{k} \frac{x^{n}}{n !} \\
B(x, t) & =\sum_{k, n \geq 0} b_{k}(n) t^{k} \frac{x^{n}}{n !} .
\end{aligned}
$$

Set $\rho=\sqrt{1-t^{2}}$. Then

$$
\begin{aligned}
& B(x, t)=\frac{2 / \rho}{1-\frac{1-\rho}{t} e^{\rho x}}-\frac{1}{\rho} \\
& A(x, t)=(1-t) B(x, t) .
\end{aligned}
$$

Many consequences can be derived from Theorem 4.2. In particular, there are explicit formulas for $a_{k}(n)$ and $b_{k}(n)$.

Corollary 4.3. For all $k, n \geq 1$ we have

$$
\begin{aligned}
& b_{k}(n)=\frac{1}{2^{k-1}} \sum_{\substack{r+2 s \leq k \\
r \equiv k(\bmod 2)}}(-2)^{s}\left(\begin{array}{c}
k-s \\
(k+r) / 2
\end{array}\right)\left(\begin{array}{l}
n \\
s
\end{array}\right) r^{n} \\
& a_{k}(n)=b_{k}(n)-b_{k-1}(n) .
\end{aligned}
$$

For $k \leq 6$ we have

$$
\begin{aligned}
& b_{2}(n)=2^{n-1} \\
& b_{3}(n)=\frac{1}{4}\left(3^{n}-2 n+3\right) \\
& b_{4}(n)=\frac{1}{8}\left(4^{n}-2(n-2) 2^{n}\right) \\
& b_{5}(n)=\frac{1}{16}\left(5^{n}-(2 n-5) 3^{n}+2\left(n^{2}-5 n+5\right)\right) \\
& b_{6}(n)=\frac{1}{32}\left(6^{n}-2(n-3) 4^{n}+\left(2 n^{2}-12 n+15\right) 2^{n}\right) .
\end{aligned}
$$

We can also obtain explicit formulas for the moments of as $(w)$. For instance, to obtain the mean (expectation)

$$
D(n)=\frac{1}{n !} \sum_{w \in \mathfrak{S}_{n}} \operatorname{as}(w)
$$


we compute

$$
\begin{aligned}
\sum_{n \geq 1} D(n) x^{n} & =\frac{\partial}{\partial t} A(x, 1) \\
& =\frac{6 x-3 x^{2}+x^{3}}{6(1-x)^{2}} \\
& =x+\sum_{n \geq 2} \frac{4 n+1}{6} x^{n} .
\end{aligned}
$$

Thus

$$
D(n)=\frac{4 n+1}{6}, \quad n \geq 2,
$$

a remarkably simple formula. Note that (not surprisingly) $D(n)$ is much larger than the expectation of is $(w)$, viz., $E(n) \sim 2 \sqrt{n}$. Similarly one can obtain explicit formulas for all the higher moments. In particular, the variance

$$
V(n)=\frac{1}{n !} \sum_{w \in \mathfrak{S}_{n}}(\operatorname{as}(w)-D(n))^{2}
$$

is given by

$$
V(n)=\frac{8}{45} n-\frac{13}{180}, \quad n \geq 4 .
$$

Now that we have computed the mean and variance of as $(w)$, we can ask whether there is an "alternating analogue" of the Baik-Deift-Johansson formula (4.2). In other words, can we determine the scaled limiting distribution

$$
K(t)=\lim _{n \rightarrow \infty} \operatorname{Prob}\left(\frac{\operatorname{as}_{n}(w)-2 n / 3}{\sqrt{n}} \leq t\right),
$$

for $t \in \mathbb{R}$ ? It turns out that the limiting distribution is Gaussian. It is a consequence of results of Pemantle and Wilson [55] and Wilf [84], and was proved directly by Widom [83]. More precisely, we have

$$
K(t)=\frac{1}{\sqrt{\pi}} \int_{-\infty}^{t \sqrt{45} / 4} e^{-s^{2}} d s .
$$

Let us mention an observation of Bóna that the statistic as $(w)$ is closely related to another statistic on permutations. Namely, an alternating run of a permutation $w \in \mathfrak{S}_{n}$ is a maximal factor (subsequence of consecutive elements) that is increasing or decreasing. For instance, the permutation 64283157 has four alternating runs, viz., $642,28,831$, and 157 . Let $g_{k}(n)$ be the number of permutations $w \in \mathfrak{S}_{n}$ with $k$ alternating runs. Then Bóna's observation $[\mathbf{1 0}]$ is that

$$
a_{k}(n)=\frac{1}{2}\left(g_{k-1}(n)+g_{k}(n)\right), \quad n \geq 2 .
$$

Hence all our results on as $(w)$ can be interpreted in terms of the number of alternating runs of $w$. For some references to work on alternating runs, see [75, $\S 4]$.

A comparison of results on is $(w)$ and as $(w)$ suggests that it might be interesting to interpolate between them. One possibility is the following. Given $k \geq 1$, define a sequence $a_{1} a_{2} \cdots a_{k}$ of integers to be $k$-alternating if

$$
a_{i}>a_{i+1} \Leftrightarrow i \equiv 1(\bmod k) \text {. }
$$


For instance, 61482573 is 3-alternating. A sequence is 2-alternating if and only if it is alternating, and a sequence of length $n$ is increasing if and only if it is $k$ alternating for some (or any) $k \geq n-1$. What is the expected value $E_{k}(n)$ and limiting distribution of the length of the longest $k$-alternating subsequence of a random permutation $w \in \mathfrak{S}_{n}$ ? If $k$ is constant then most likely $E_{k}(n) \sim c_{k} n$ for some constant $c_{k}$, while the limiting distribution remains Gaussian (as for $k=2$ ). But what if $k$ grows with $n$, e.g., $k=\lfloor\sqrt{n}\rfloor$ ? Is there a sharp cutoff between the behavior $E_{k}(n) \sim c n$ and $E_{k}(n) \sim c \sqrt{n}$, or is there a wide range of intermediate values? Similarly, do we get limiting distributions other than Gaussian or TracyWidom? The same questions can be asked if we replace $k$-alternating with the condition that $a_{i}>a_{i+1}$ if and only if $\lfloor i / k\rfloor$ is even, i.e., the permutation begins with $k-1$ descents, then $k-1$ ascents, etc.

\section{Umbral enumeration of classes of alternating permutations}

In this section we consider the enumeration of alternating permutations having additional properties, such as having alternating inverses or having no fixed points. The main tool will be a certain character $\chi^{\tau_{n}}$ of the symmetric group $\mathfrak{S}_{n}$, first considered by H. O. Foulkes [28][29], whose dimension in $E_{n}$. For the definition of $\chi^{\tau_{n}}$ we assume familiarity with the theory of symmetric functions as in Section 3.6.

For any skew shape $\lambda / \mu$ of size $n$ we can associate a character $\chi^{\lambda / \mu}$ of $\mathfrak{S}_{n}$, e.g., by letting the Frobenius characteristic $\operatorname{ch}\left(\chi^{\lambda / \mu}\right)$ be the skew Schur function $s_{\lambda / \mu}$. In particular, taking $\tau_{n}$ to be the zigzag shape of Section 3.6 gives the character $\chi^{\tau_{n}}$. For any character $\chi$ of $\mathfrak{S}_{n}$ and partition $\mu$ of $n$ we write $\chi(\mu)$ for $\chi(w)$, where $w$ is a permutation of cycle type $\mu$. The main result [29, Thm. 6.1][72, Exer. 7.64] of Foulkes on the connection between alternating permutations and representation theory is the following.

ThEOREM 5.1. (a) Let $\mu \vdash n$, where $n=2 k+1$. Then

$$
\chi^{\tau_{n}}(\mu)=\chi^{\tau_{n}^{\prime}}(\mu)=\left\{\begin{array}{cc}
0, & \text { if } \mu \text { has an even part } \\
(-1)^{k+r} E_{2 r+1}, & \text { if } \mu \text { has } 2 r+1 \text { odd parts and } \\
\text { no even parts. }
\end{array}\right.
$$

(b) Let $\mu \vdash n$, where $n=2 k$. Suppose that $\mu$ has $2 r$ odd parts and e even parts. Then

$$
\begin{aligned}
\chi^{\tau_{n}}(\mu) & =(-1)^{k+r+e} E_{2 r} \\
\chi^{\tau_{n}^{\prime}}(\mu) & =(-1)^{k+r} E_{2 r} .
\end{aligned}
$$

Foulkes' result leads immediately to the main tool of this section. We will use umbral notation [61] for Euler numbers. In other words, any polynomial in $E$ is to be expanded in terms of powers of $E$, and then $E^{k}$ is replaced by $E_{k}$. The replacement of $E^{k}$ by $E_{k}$ is always the last step in the evaluation of an umbral expression. For instance,

$$
\left(E^{2}-1\right)^{2}=E^{4}-2 E^{2}+1=E_{4}-2 E_{2}+1=5-2 \cdot 1+1=4 .
$$


Similarly,

$$
\begin{aligned}
(1+t)^{E} & =1+E t+\left(\begin{array}{c}
E \\
2
\end{array}\right) t^{2}+\left(\begin{array}{c}
E \\
3
\end{array}\right) t^{3}+\cdots \\
& =1+E t+\frac{1}{2}\left(E^{2}-E\right) t^{2}+\frac{1}{6}\left(E^{3}-3 E^{2}+2 E\right) t^{3}+\cdots \\
& =1+E_{1} t+\frac{1}{2}\left(E_{2}-E_{1}\right) t^{2}+\frac{1}{6}\left(E_{3}-3 E_{2}+2 E_{1}\right) t^{3}+\cdots \\
& =1+1 \cdot t+\frac{1}{2}(1-1) t^{2}+\frac{1}{6}(2-3 \cdot 1+2 \cdot 1) t^{3}+\cdots \\
& =1+t+\frac{1}{6} t^{3}+\cdots
\end{aligned}
$$

If $f=f\left(x_{1}, x_{2}, \ldots\right)$ is a symmetric function then we use the notation $f\left[p_{1}, p_{2}, \ldots\right]$ for $f$ regarded as a polynomial in the power sums. For instance, if $f=e_{2}=$ $\sum_{i<j} x_{i} x_{j}=\frac{1}{2}\left(p_{1}^{2}-p_{2}\right)$ then

$$
e_{2}[E,-E, \ldots]=\frac{1}{2}\left(E^{2}+E\right)=1 .
$$

We also write $\langle f, g\rangle$ for the standard (Hall) scalar product of the symmetric functions $f$ and $g$.

THEOREM 5.2. Let $f$ be a homogenous symmetric function of degree $n$. If $n$ is odd then

$$
\left\langle f, s_{\tau_{n}}\right\rangle=\left\langle f, s_{\tau_{n}^{\prime}}\right\rangle=f[E, 0,-E, 0, E, 0,-E, \ldots]
$$

If $n$ is even then

$$
\begin{aligned}
\left\langle f, s_{\tau_{n}}\right\rangle & =f[E,-1,-E, 1, E,-1,-E, 1, \ldots] \\
\left\langle f, s_{\tau_{n}^{\prime}}\right\rangle & =f[E, 1,-E,-1, E, 1,-E,-1, \ldots] .
\end{aligned}
$$

There are numerous results in the theory of symmetric functions that express the number of permutations with certain properties as scalar products of symmetric functions. Using Theorem 5.2 we can obtain umbral generating functions for the number of alternating permutations with various properties. Sometimes it is possible to "deumbralize" the generating function by expanding it explicitly in powers of $E$ and then replacing $E^{k}$ with $E_{k}$.

As an example of the procedure described above, let $f(n)$ denote the number of permutations $w \in \mathfrak{S}_{n}$ such that both $w$ and $w^{-1}$ are alternating. Such permutations are called doubly alternating. As a special case of a well-known result of Foulkes [28, Thm. 6.2] (see also [72, Cor. 7.23.8]), we have

$$
f(n)=\left\langle s_{\tau_{n}}, s_{\tau_{n}}\right\rangle .
$$

We can now use Theorem 5.2 to obtain (see [74] for details)

$$
\begin{aligned}
\sum_{k \geq 0} f(2 k+1) t^{2 k+1} & =\sum_{r \geq 0} E_{2 r+1}^{2} \frac{L(t)^{2 r+1}}{(2 r+1) !} \\
\sum_{k \geq 0} f(2 k) t^{2 k} & =\frac{1}{\sqrt{1-t^{2}}} \sum_{r \geq 0} E_{2 r}^{2} \frac{L(t)^{2 r}}{(2 r) !},
\end{aligned}
$$


where

$$
L(t)=\frac{1}{2} \log \frac{1+t}{1-t}=t+\frac{t^{3}}{3}+\frac{t^{5}}{5}+\cdots .
$$

A further class of alternating permutations that can be enumerated by the above technique are those of a given cycle type, i.e., with specified cycle lengths in their representation as a product of disjoint cycles. We will only mention two special cases here. The first case is when all cycles have length two. Such permutations are just the fixed-point-free involutions. They necessarily have even length. Thus let $g(n)$ (respectively, $g^{*}(n)$ ) denote the number of fixed-point-free alternating involutions (respectively, fixed-point-free reverse alternating involutions) in $\mathfrak{S}_{2 n}$. Set

$$
\begin{aligned}
& G(t)=\sum_{n \geq 0} g(n) x^{n}=1+t+t^{2}+2 t^{3}+5 t^{4}+17 t^{5}+72 t^{6}+\cdots \\
& G^{*}(t)=\sum_{n \geq 0} g^{*}(n) x^{n}=1+t^{2}+t^{3}+4 t^{4}+13 t^{5}+59 t^{6}+\cdots .
\end{aligned}
$$

THEOREM 5.3. We have the umbral generating functions

$$
\begin{aligned}
G(t) & =\left(\frac{1+t}{1-t}\right)^{\left(E^{2}+1\right) / 4} \\
G^{*}(t) & =\frac{G(t)}{1+t}
\end{aligned}
$$

Ramanujan asserts on page 324 of his second notebook (see [8, p. 545]) that as $t$ tends to $0+$,

$$
2 \sum_{n \geq 0}(-1)^{n}\left(\frac{1-t}{1+t}\right)^{n(n+1)} \sim 1+t+t^{2}+2 t^{3}+5 t^{4}+17 t^{5}+\cdots .
$$

Berndt $[8,(16.6)]$ obtains a formula for the complete asymptotic expansion of $2 \sum_{n \geq 0}(-1)^{n}\left(\frac{1-t}{1+t}\right)^{n(n+1)}$ as $t \rightarrow 0+$. It is easy to see that Berndt's formula can be written as $\left(\frac{1+t}{1-t}\right)^{\left(E^{2}+1\right) / 4}$ and is thus equal to $G(t)$. Theorem 5.3 therefore answers a question of Galway [32, p. 111], who asks for a combinatorial interpretation of the coefficients in Ramanujan's asymptotic expansion. Is there a direct way to see the connection between equation (5.2) and fixed-point-free alternating involutions? Can equation (5.2) be generalized to involve other classes of permutations?

Note. The following formula for $G(t)$ follows from equation (5.2) and an identity of Ramanujan proved by Andrews $\left[\mathbf{2},(6.3)_{\mathrm{R}}\right]$ :

$$
F_{2}(t)=2 \sum_{n \geq 0} q^{n} \frac{\prod_{j=1}^{n}\left(1-q^{2 j-1}\right)}{\prod_{j=1}^{2 n+1}\left(1+q^{j}\right)}
$$

where $q=\left(\frac{1-t}{1+t}\right)^{2 / 3}$. It is not hard to see that this is a formal identity, unlike the asymptotic identity (5.2).

The second class of alternating permutations with a given cycle type that we will discuss are those that are single cycles. Let $b(n)$ (respectively, $\left.b^{*}(n)\right)$ denote the number of alternating (respectively, reverse alternating) $n$-cycles in $\mathfrak{S}_{n}$.

THEOREM 5.4. (a) If $n$ is odd then

$$
b(n)=b^{*}(n)=\frac{1}{n} \sum_{d \mid n} \mu(d)(-1)^{(d-1) / 2} E_{n / d} .
$$


(b) If $n=2^{k} m$ where $k \geq 1$, $m$ is odd, and $m \geq 3$, then

$$
b(n)=b^{*}(n)=\frac{1}{n} \sum_{d \mid m} \mu(d) E_{n / d} .
$$

(c) If $n=2^{k}$ and $k \geq 2$ then

$$
b(n)=b^{*}(n)=\frac{1}{n}\left(E_{n}-1\right) .
$$

(d) Finally, $b(2)=1, b^{*}(2)=0$.

Note that curious fact that $b(n)=b^{*}(n)$ except for $n=2$. Can Theorem 5.4 be proved combinatorially, especially in the cases when $n$ is a prime power (when the sums have only two terms)?

It is immediate from Theorem 5.4 that $\lim _{n \rightarrow \infty} n b(n) / E_{n}=1$. Thus as $n \rightarrow$ $\infty$, a fraction $1 / n$ of the alternating permutations are $n$-cycles. Compare this with the simple fact that (exactly) $1 / n$ of the permutations $w \in \mathfrak{S}_{n}$ are $n$-cycles. We can say that the properties of being an alternating permutation and an $n$ cycle are "asymptotically independent." What other class of permutations are asymptotically independent from the alternating permutations?

Closely related to the cycle types of alternating permutations is the enumeration of alternating permutations with a given number of fixed points, a question first raised by P. Diaconis. Thus let $d_{k}(n)$ (respectively, $d_{k}^{*}(n)$ ) denote the number of alternating (respectively, reverse alternating) permutations in $\mathfrak{S}_{n}$ with exactly $k$ fixed points. For a power series $F(t)=\sum a_{n} t^{n}$ we write

$$
\begin{aligned}
& \mathcal{O}_{t} F(t)=\frac{1}{2}(F(t)-F(-t))=\sum a_{2 n+1} t^{2 n+1} \\
& \mathcal{E}_{t} F(t)=\frac{1}{2}(F(t)+F(-t))=\sum a_{2 n} t^{2 n}
\end{aligned}
$$

THEOREM 5.5. We have

$$
\begin{aligned}
\sum_{k, n \geq 0} d_{k}(2 n+1) q^{k} t^{2 n+1} & =\mathcal{O}_{t} \frac{\exp \left(E\left(\tan ^{-1} q t-\tan ^{-1} t\right)\right)}{1-E t} \\
d_{k}^{*}(2 n+1) & =d_{k}(2 n+1) \\
\sum_{k, n \geq 0} d_{k}(2 n) q^{k} t^{2 n} & =\mathcal{E}_{t} \sqrt{\frac{1+t^{2}}{1+q^{2} t^{2}}} \frac{\exp \left(E\left(\tan ^{-1} q t-\tan ^{-1} t\right)\right)}{1-E t} \\
\sum_{k, n \geq 0} d_{k}^{*}(2 n) q^{k} t^{2 n} & =\mathcal{E}_{t} \sqrt{\frac{1+q^{2} t^{2}}{1+t^{2}}} \frac{\exp \left(E\left(\tan ^{-1} q t-\tan ^{-1} t\right)\right)}{1-E t}
\end{aligned}
$$


Equivalently, we have the non-umbral formulas

$$
\begin{aligned}
\sum_{k, n \geq 0} d_{k}(2 n+1) q^{k} t^{2 n+1} & =\sum_{\substack{i, j \geq 0 \\
i \neq j(\bmod 2)}} \frac{E_{i+j}}{j !} t^{i}\left(\tan ^{-1} q t-\tan ^{-1} t\right)^{j} \\
\sum_{k, n \geq 0} d_{k}(2 n) q^{k} t^{2 n} & =\sqrt{\frac{1+t^{2}}{1+q^{2} t^{2}}} \sum_{\substack{i, j \geq 0 \\
i \equiv j(\bmod 2)}} \frac{E_{i+j}}{j !} t^{i}\left(\tan ^{-1} q t-\tan ^{-1} t\right)^{j} \\
\sum_{k, n \geq 0} d_{k}^{*}(2 n) q^{k} t^{2 n} & =\sqrt{\frac{1+q^{2} t^{2}}{1+t^{2}}} \sum_{\substack{i, j \geq 0 \\
i \equiv j(\bmod 2)}} \frac{E_{i+j}}{j !} t^{i}\left(\tan ^{-1} q t-\tan ^{-1} t\right)^{j}
\end{aligned}
$$

Theorem 5.5 may look a little daunting, but it can be used to obtain some interesting concrete results. For instance, it is not difficult to deduce from Theorem 5.5 that $d_{0}(n)=d_{1}(n)$ and $d_{0}^{*}(n)=d_{1}^{*}(n)$, yet another result that cries out for a combinatorial proof. Moreover, we can obtain an asymptotic expansion for the number of alternating derangements (permutations without fixed points) in $\mathfrak{S}_{n}$, as follows.

Corollary 5.6. (a) We have for $n$ odd the asymptotic expansion

$$
\begin{aligned}
d_{0}(n) & \sim \frac{1}{e}\left(E_{n}+a_{1} E_{n-2}+a_{2} E_{n-4}+\cdots\right) \\
& =\frac{1}{e}\left(E_{n}+\frac{1}{3} E_{n-2}-\frac{13}{90} E_{n-4}+\frac{467}{5760} E_{n-6}+\cdots\right),
\end{aligned}
$$

where

$$
\sum_{k \geq 0} a_{k} x^{2 k}=\exp \left(1-\frac{1}{x} \tan ^{-1} x\right) .
$$

(b) We have for $n$ even the asymptotic expansion

$$
\begin{aligned}
d_{0}(n) & \sim \frac{1}{e}\left(E_{n}+b_{1} E_{n-2}+b_{2} E_{n-4}+\cdots\right) \\
& =\frac{1}{e}\left(E_{n}+\frac{5}{6} E_{n-2}-\frac{37}{360} E_{n-4}+\frac{281}{9072} E_{n-6}+\cdots\right),
\end{aligned}
$$

where

$$
\sum_{k \geq 0} b_{k} x^{2 k}=\sqrt{1+x^{2}} \exp \left(1-\frac{1}{x} \tan ^{-1} x\right) .
$$

(c) We have for $n$ even the asymptotic expansion

$$
\begin{aligned}
d_{0}^{*}(n) & \sim \frac{1}{e}\left(E_{n}+c_{1} E_{n-2}+c_{2} E_{n-4}+\cdots\right) \\
& =\frac{1}{e}\left(E_{n}-\frac{1}{6} E_{n-2}+\frac{23}{360} E_{n-4}-\frac{1493}{45360} E_{n-6}+\cdots\right),
\end{aligned}
$$

where

$$
\sum_{k \geq 0} c_{k} x^{2 k+1}=\frac{1}{\sqrt{1+x^{2}}} \exp \left(1-\frac{1}{x} \tan ^{-1} x\right) .
$$


If $D(n)$ denotes the total number of derangements in $\mathfrak{S}_{n}$, then it is a basic result from enumerative combinatorics that $\lim _{n \rightarrow \infty} d(n) / n !=1 / e$. On the other hand, Corollary 5.6 shows that $\lim _{n \rightarrow \infty} d_{0}(n) / E_{n}=\lim _{n \rightarrow \infty} d_{0}^{*}(n) / E_{n}=1 / e$. In other words, the property of being a derangement is asymptotically independent from being an alternating permutation or from being a reverse alternating permutation.

A further consequence of Theorem 5.5 concerns the total number of fixed points of alternating and reverse alternating permutations in $\mathfrak{S}_{n}$. Again it is instructive to compare with the case of all permutations. It is an immediate consequence of Burnside's lemma (also known as the Cauchy-Frobenius lemma) and is easy to see combinatorially that the total number of fixed points of all permutations $w \in \mathfrak{S}_{n}$ is $n$ !. For alternating permutations we have the following result.

ThEOREM 5.7. Let $f(n)$ (respectively, $g(n)$ ) denote the total number of fixed points of alternating (respectively, reverse alternating) permutations in $\mathfrak{S}_{n}$. Then

$$
\begin{gathered}
f(n)=\left\{\begin{array}{cc}
E_{n}-E_{n-2}+E_{n-4}-\cdots+(-1)^{(n-1) / 2} E_{1}, & n \text { odd } \\
E_{n}-2 E_{n-2}+2 E_{n-4}-\cdots+(-1)^{(n-2) / 2} 2 E_{2}+(-1)^{n / 2}, & n \text { even. }
\end{array}\right. \\
g(n)=\left\{\begin{array}{cl}
E_{n}-E_{n-2}+E_{n-4}-\cdots+(-1)^{(n-1) / 2} E_{1}, & n \text { odd } \\
E_{n}-(-1)^{n / 2}, & n \text { even. }
\end{array}\right.
\end{gathered}
$$

Can these results be proved combinatorially?

There are umbral formulas for enumerating alternating and reverse alternating permutations according to the length of their longest increasing subsequence. These results are alternating analogues of the following result of Gessel $[\mathbf{3 4}$, p. 280]. Let $u_{k}(n)$ be the number of permutations $w \in \mathfrak{S}_{n}$ whose longest increasing subsequence has length at most $k$. For instance, $u_{2}(n)=C_{n}$, a Catalan number $[\mathbf{7 2}$, Exer. 6.19(ee)]. Define

$$
\begin{aligned}
& U_{k}(x)=\sum_{n \geq 0} u_{k}(n) \frac{x^{2 n}}{n !^{2}}, k \geq 1 \\
& I_{i}(2 x)=\sum_{n \geq 0} \frac{x^{2 n+i}}{n !(n+i) !}, i \geq 0 .
\end{aligned}
$$

The function $I_{i}$ is the hyperbolic Bessel function of the first kind of order $i$. Gessel then showed that

$$
U_{k}(x)=\operatorname{det}\left(I_{|i-j|}(2 x)\right)_{i, j=1}^{k} .
$$

We state without proof the alternating analogue of equation (5.4). It is proved by applying the umbral techniques of $[\mathbf{7 4}]$ to a symmetric function identity $[\mathbf{3 4}$, Thm. 16] of Gessel. For any integer $i$ we use the notation $D^{i} F(x)$ for the $n$th formal derivative of the power series $F(x)=\sum_{n \geq 0} a_{n} x^{n}$, where in particular

$$
D^{-1} F(x)=\sum_{n \geq 0} a_{n} \frac{x^{n+1}}{n+1},
$$

and $D^{-i-1}=D^{-1} D^{-i}$ for all $i \geq 1$.

THEOREM 5.8. Let $v_{k}(n)$ (respectively, $v_{k}^{\prime}(n)$ ) denote the number of alternating (respectively, reverse alternating) permutations $w \in \mathfrak{S}_{n}$ whose longest increasing 
subsequence has length at most $n$. Let $\exp \left(E \tan ^{-1}(x)\right)=\sum_{n \geq 0} c_{n}(E) x^{n}$. Define

$$
\begin{aligned}
& A_{1}(x)=\sum_{n \geq 0} c_{n}(E) \frac{x^{n}}{n !} \\
& A_{2}(x)=\sqrt{1+x^{2}} A_{1}(x) \\
& A_{3}(x)=\frac{A_{1}(x)}{\sqrt{1+x^{2}}},
\end{aligned}
$$

and for $1 \leq r \leq 3$ and $k \geq 1$ define

$$
B_{r, k}(x)=\operatorname{det}\left(D^{j-i} A_{r}(x)\right)_{i, j=1}^{k} .
$$

We then have:

- If $n$ is odd, then the coefficient of $x^{n} / n$ ! in the umbral evaluation of $B_{1, k}(x)$ is $v_{k}(n)=v_{k}^{\prime}(n)$.

- If $n$ is even, then the coefficient of $x^{n} / n$ ! in the umbral evaluation of $B_{2, k}(x)$ is $v_{k}(n)$.

- If $n$ is even, then the coefficient of $x^{n} / n$ ! in the umbral evaluation of $B_{3, k}(x)$ is $v_{k}^{\prime}(n)$.

Note that it follows from Theorem 5.8 that $v_{k}(2 m+1)=v_{k}^{\prime}(2 m+1)$. This result is also an immediate consequence of the involution (1.1) followed by reversal of the permutation.

It is known (see [76]) that $v_{2}(n)=C_{\lceil(n+1) / 2\rceil}$ for $n \geq 3$ and $v_{2}^{\prime}(n)=C_{\lceil n / 2\rceil}$ for $n \geq 1$, where $C_{i}=\frac{1}{i+1}\left(\begin{array}{c}2 i \\ i\end{array}\right)$ (a Catalan number). Can these formulas be deduced directly from the case $k=2$ of Theorem 5.8? Similarly, J. Lewis [47] has shown that

$$
v_{3}^{\prime}(n)=\left\{\begin{aligned}
f^{(m, m, m)}, & n=2 m \\
f^{(m-1, m, m+1)}, & n=2 m+1,
\end{aligned}\right.
$$

No such results are known for $v_{4}(n)$ or $v_{3}(n)$.

Is there an asymptotic formula for the expected length of the longest increasing subsequence of an alternating permutation $w \in \mathfrak{S}_{n}$ as $n \rightarrow \infty$, analogous to the result (4.1) of Logan-Shepp and Vershik-Kerov for arbitrary permutations? Or more strongly, is there a limiting distribution for the (suitably scaled) length of the longest increasing subsequence of an alternating permutation $w \in \mathfrak{S}_{n}$ as $n \rightarrow \infty$, analogous to the Baik-Deift-Johansson theorem (4.2) for arbitrary permutations? Two closely related problems are the following. Let $a^{\lambda}$ denote the number of SYT of shape $\lambda \vdash n$ and descent set $\{1,3,5, \ldots\} \cap[n-1]$, as defined in [72, p. 361]. (These are just the SYT $Q$ that arise from alternating permutations $w \in \mathfrak{S}_{n}$ by applying the RSK algorithm $w \mapsto(P, Q)$.) Let $f^{\lambda}$ denote the number of SYT of shape $\lambda$, given by the famous hook length formula [72, Cor. 7.21.6]. What is the (suitably scaled) limiting shape of $\lambda$ as $n \rightarrow \infty$ that maximizes $a^{\lambda}$ and similarly that maximizes $a^{\lambda} f^{\lambda}$ ? (For the shape that maximizes $f^{\lambda}$, see $[\mathbf{7 3}, \S 3]$. Could this shape also maximize $a^{\lambda}$ ?)

Note. Non-umbral methods have been used to enumerate certain classes of (reverse) alternating permutations. A Baxter permutation (originally called a reduced Baxter permutation) is a permutation $w \in \mathfrak{S}_{n}$ satisfying: if $w(r)=i$ and $w(s)=i+1$, then there is a $k_{i}$ between $r$ and $s$ (i.e., $r \leq k_{i} \leq s$ or $s \leq k_{i} \leq r$ ) such that $w(t) \leq i$ if $t$ is between $r$ and $k_{i}$, while $w(t) \geq i+1$ if $t$ is between $k_{i}+1$ and $s$. For instance, all permutations $w \in \mathfrak{S}_{4}$ are Baxter permutations except 2413 
and 3142. Cori, Dulucq, and Viennot [15] showed that the number of reverse alternating Baxter permutations in $\mathfrak{S}_{2 n}$ is $C_{n}^{2}$, and in $\mathfrak{S}_{2 n+1}$ is $C_{n} C_{n+1}$. Guibert and Linusson [39] showed that the number of reverse alternating Baxter permutations $\mathfrak{S}_{n}$ whose inverses are also reverse alternating is just $C_{n}$. A different proof was later given by Min and Park [52]. Some related work on enumerating special classes of alternating permutations was done by Dulucq and Simion [19]. A popular topic in permutation enumeration is pattern avoidance. See $[\mathbf{9}$, Ch. 5] for an introduction. A special case deals with permutations with no increasing subsequence of length $k$. Mansour [50] inaugurated the study of pattern avoidance in alternating permutations. For some additional papers on this topic see Hong $[\mathbf{4 2}]$, Lewis $[\mathbf{4 5}][\mathbf{4 7}]$, and Ouchterlony [54].

\section{The $c d$-index of $\mathfrak{S}_{n}$}

Let $w=a_{1} \cdots a_{n} \in \mathfrak{S}_{n}$. The descent set $D(w)$ of $w$ is defined by

$$
D(w)=\left\{i: a_{i}>a_{i+1}\right\} \subseteq[n-1] .
$$

A permutation $w$ is thus alternating if $D(w)=\{1,3,5, \ldots\} \cap[n-1]$ and reverse alternating if $D(w)=\{2,4,6, \ldots\} \cap[n-1]$. For $S \subseteq[n-1]$ let

$$
\beta_{n}(S)=\#\left\{w \in \mathfrak{S}_{n}: D(w)=S\right\} .
$$

The numbers $\beta_{n}(S)$ are fundamental invariants of $\mathfrak{S}_{n}$ that appear in a variety of combinatorial, algebraic, and geometric contexts. In this section we explain how alternating permutations and Euler numbers are related to the more general subject of permutations with a fixed descent set.

We first define for fixed $n$ a noncommutative generating function for the numbers $\beta_{n}(S)$. Given a set $S \subseteq[n-1]$, define its characteristic monomial (or variation) to be the noncommutative monomial

$$
u_{S}=e_{1} e_{2} \cdots e_{n-1}
$$

where

$$
e_{i}= \begin{cases}a, & \text { if } i \notin S \\ b, & \text { if } i \in S .\end{cases}
$$

For instance, $D(37485216)=\{2,4,5,6\}$, so $u_{D(37485216)}=a b a b b b a$. Define

$$
\begin{aligned}
\Psi_{n}=\Psi_{n}(a, b) & =\sum_{w \in \mathfrak{S}_{n}} u_{D(w)} \\
& =\sum_{S \subseteq[n-1]} \beta_{n}(S) u_{S} .
\end{aligned}
$$

Thus $\Psi_{n}$ is a noncommutative generating function for the numbers $\beta_{n}(S)$. For instance,

$$
\begin{aligned}
& \Psi_{1}=1 \\
& \Psi_{2}=a+b \\
& \Psi_{3}=a^{2}+2 a b+2 b a+b^{2} \\
& \Psi_{4}=a^{3}+3 a^{2} b+5 a b a+3 b a^{2}+3 a b^{2}+5 b a b+3 b^{2} a+b^{3} .
\end{aligned}
$$

The polynomial $\Psi_{n}$ is called the $a b$-index of the symmetric group $\mathfrak{S}_{n}$.

The main result of this section is the following. 


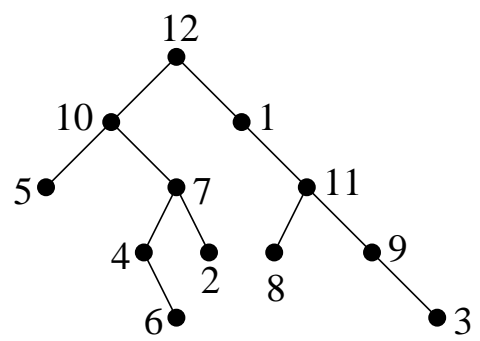

(a)

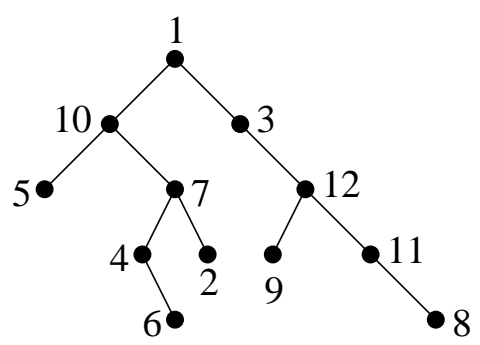

(b)

Figure 7. (a) The min-max tree $M=$ $M(5,10,4,6,7,2,12,1,8,11,9,3) ; \quad$ (b) The transformed tree $\psi_{7} M=M(5,10,4,6,7,2,1,3,9,12,11,8)$

THEOREM 6.1. There exists a polynomial $\Phi_{n}(c, d)$ in the noncommuting variables $c$ and $d$ such that

$$
\Psi_{n}(a, b)=\Phi_{n}(a+b, a b+b a) .
$$

The polynomial $\Phi_{n}(c, d)$ is called the $c d$-index of $\mathfrak{S}_{n}$. For instance, we have

$$
\Psi_{3}(a, b)=a^{2}+2 a b+2 b a+b^{2}=(a+b)^{2}+(a b+b a),
$$

so $\Phi_{3}(c, d)=c^{2}+d$. Some values of $\Phi_{n}(c, d)$ for small $n$ are as follows:

$$
\begin{aligned}
& \Phi_{1}=1 \\
& \Phi_{2}=c \\
& \Phi_{3}=c^{2}+d \\
& \Phi_{4}=c^{3}+2 c d+2 d c \\
& \Phi_{5}=c^{4}+3 c^{2} d+5 c d c+3 d c^{2}+4 d^{2} \\
& \Phi_{6}=c^{5}+4 c^{3} d+9 c^{2} d c+9 c d c^{2}+4 d c^{3}+12 c d^{2}+10 d c d+12 d^{2} c
\end{aligned}
$$

If we define $\operatorname{deg}(c)=1$ and $\operatorname{deg}(d)=2$, then the number of $c d$-monomials of degree $n-1$ is the Fibonacci number $F_{n}$. All these monomials actually appear in $\Phi_{n}(c, d)$ (as in evident from the discussion below). Thus $\Phi_{n}(c, d)$ has $F_{n}$ terms, compared with $2^{n-1}$ terms for $\Psi_{n}(a, b)$.

There are several known proofs of Theorem 6.1. Perhaps the most natural approach is to define an equivalence relation on $\mathfrak{S}_{n}$ such that for each equivalence class $C$, we have that $\sum_{w \in C} u_{D(w)}$ is a monomial in $c=a+b$ and $d=a b+b a$. Such a proof was given by G. Hetyei and E. Reiner [41]. We will simply define the equivalence relation here. An exposition appears in Stanley [71, §1.6].

We first define the min-max tree $M(w)$ associated with a sequence $w=a_{1} a_{2} \cdots a_{n}$ of distinct integers as follows. First, $M(w)$ is a binary tree with vertices labelled $a_{1}, a_{2}, \ldots, a_{n}$. Let $j$ be the least integer for which either $a_{j}=\min \left\{a_{1}, \ldots, a_{n}\right\}$ or $a_{j}=\max \left\{a_{1}, \ldots, a_{n}\right\}$. Define $a_{j}$ to be the root of $M(w)$. Then define (recursively) $M\left(a_{1}, \ldots, a_{j-1}\right)$ to be the left subtree of $a_{j}$, and $M\left(a_{j+1}, \ldots, a_{n}\right)$ to be the right subtree. Figure $7(\mathrm{a})$ shows $M(5,10,4,6,7,2,12,1,8,11,9,3)$. Note that no vertex of a min-max tree $M(w)$ has only a left successor. Note also that every vertex $v$ is either the minimum or maximum element of the subtree with root $v$. 
Given the min-max tree $M(w)$ where $w=a_{1} \cdots a_{n}$, we will define operators $\psi_{i}$, $1 \leq i \leq n$, that permute the labels of $M(w)$, creating a new min-max tree $\psi_{i} M(w)$. The operator $\psi_{i}$ only permutes the label of the vertex of $M(w)$ labelled $a_{i}$ and the labels of the right subtree of this vertex. (Note that the vertex labelled $a_{i}$ depends only on $i$ and the tree $M(w)$, not on the permutation $w$.) All other vertices are fixed by $\psi_{i}$. In particular, if $a_{i}$ is an endpoint then $\psi_{i} M(w)=M(w)$. Suppose that $a_{i}$ is the minimum element of the subtree $M_{a_{i}}$ with root $a_{i}$. Then replace $a_{i}$ with the largest element of $M_{a_{i}}$, and permute the remaining elements of $M_{a_{i}}$ so that they keep their same relative order. This defines $\psi_{i} M(w)$. Similarly suppose that $a_{i}$ is the maximum element of the subtree $M_{a_{i}}$ with root $a_{i}$. Then replace $a_{i}$ with the smallest element of $M_{a_{i}}$, and permute the remaining elements of $M_{a_{i}}$ so that they keep their same relative order. Again this defines $\psi_{i} M(w)$. Figure $7(\mathrm{~b})$ shows that $\psi_{7} M(5,10,4,6,7,2,12,1,8,11,9,3)=M(5,10,4,6,7,2,1,3,9,12,11,8)$. We have $a_{7}=12$, so $\psi_{7}$ permutes vertex 12 and the vertices on the right subtree of 12 . Vertex 12 is replaced by 1 , the smallest vertex of the right subtree. The remaining elements $1,3,8,9,11$ get replaced with $3,8,9,11,12$ in that order.

Let us call two permutations $v, w \in \mathfrak{S}_{n}$ equivalent, denoted $v \stackrel{M}{\sim} w$, if their minmax trees $T(v)$ and $T(w)$ can be obtained from each other by applying a sequence of $\psi_{i}$ 's. Clearly $\stackrel{M}{\sim}$ is an equivalence relation on $\mathfrak{S}_{n}$. Let $c, d, e$ be noncommutative indeterminates, and let $w=a_{1} a_{2} \cdots a_{n} \in \mathfrak{S}_{n}$. For $1 \leq i \leq n$ define

$$
f_{i}=f_{i}(w)= \begin{cases}c, & \text { if } a_{i} \text { has only a right child in } M(w) \\ d, & \text { if } a_{i} \text { has a left and right child } \\ e, & \text { if } a_{i} \text { is an endpoint. }\end{cases}
$$

Let $\Phi_{w}^{\prime}=\Phi_{w}^{\prime}(c, d, e)=f_{1} f_{2} \cdots f_{n}$, and let $\Phi_{w}=\Phi_{w}(c, d)=\Phi^{\prime}(c, d, 1)$, where 1 denotes the empty word. In other words, $\Phi_{w}$ is obtained from $\Phi_{w}^{\prime}$ by deleting the $e$ 's. For instance, consider the permutation $w=5,10,4,6,7,2,12,1,8,11,9,3$ of Figure 7. The degrees (number of children) of the vertices $a_{1}, a_{2}, \ldots, a_{12}$ are 0,2 , $1,0,2,0,2,1,0,2,1,0$, respectively. Hence

$$
\begin{aligned}
& \Phi_{w}^{\prime}=\text { edcededcedce } \\
& \Phi_{w}=d c d d c d c .
\end{aligned}
$$

It is clear that if $v \stackrel{M}{\sim} w$, then $\Phi_{v}^{\prime}=\Phi_{w}^{\prime}$ and $\Phi_{v}=\Phi_{w}$, since $\Phi_{w}^{\prime}$ depends only on $M(w)$ regarded as an unlabelled tree.

The main result on min-max trees is the following.

TheOREM 6.2. For any $w \in \mathfrak{S}_{n}$ we have

$$
\sum_{\substack{M \\ v \sim w}} u_{D(v)}=\Phi_{w}(a+b, a b+b a) .
$$

Theorem 6.2 shows that $\stackrel{M}{\sim}$ is precisely the equivalence relation we asked for in order to prove the existence of the $c d$-index $\Phi_{n}(c, d)$ (Theorem 6.1). Thus not only have we shown the existence of $\Phi_{n}(c, d)$, but also we have shown that the coefficients are nonnegative. It is reasonable to ask whether there is a more "direct" description of the coefficients. Such a description was first given by D. Foata and M.-P. Schützenberger [26] in terms of the André permutations mentioned in Section 3.4. We state here the analogous result for simsun permutations (as defined in Section 3.4), due to R. Simion and S. Sundaram. 
TheOREm 6.3. Let $\mu$ be a monomial of degree $n-1$ in the noncommuting variables $c, d$, where $\operatorname{deg}(c)=1$ and $\operatorname{deg}(d)=2$. Replace each $c$ in $\mu$ with 0 , each $d$ with 10, and remove the final 0 . We get the characteristic vector of a set $S_{\mu} \subseteq[n-2]$. Then the coefficient of $\mu$ in $\Phi_{n}(c, d)$ is equal to the number of simsun permutations in $\mathfrak{S}_{n-1}$ with descent set $S_{\mu}$.

For example, if $\mu=c d^{2} c^{2} d$ then we get the characteristic vector 01010001 of the set $S_{\mu}=\{2,4,8\}$. Hence the coefficient of $c d^{2} c^{2} d$ in $\Phi_{10}(c, d)$ is equal to the number of simsun permutations in $\mathfrak{S}_{9}$ with descent set $\{2,4,8\}$.

Note that every $c d$-monomial, when expanded in terms of $a b$-monomials, is a sum of distinct monomials including bababa $\cdots$ and $a b a b a b \cdots$. These monomials correspond to descent sets of alternating and reverse alternating permutations, respectively. Hence $\Phi_{n}(1,1)=E_{n}$. This fact also follows immediately from Theorems 3.3 and 6.3.

Extending the reasoning of the previous paragraph gives a nice result on inequalities among the numbers $\beta_{n}(S)$, originally due to Niven [53] and de Bruijn [11] by different methods. The proof given below first appeared in Stanley [69, Thm. 2.3(b)] in a more general context.

Given $S \subseteq[n-1]$, define $\omega(S) \subseteq[n-2]$ by the condition $i \in \omega(S)$ if and only if exactly one of $i$ and $i+1$ belongs to $S$, for $1 \leq i \leq n-2$. For instance, if $n=9$ and $S=\{2,4,5,8\}$, then $\omega(S)=\{1,2,3,5,7\}$. Note that

Proposition 6.4. Let $S, T \subseteq[n-1]$. If $\omega(S) \subset \omega(T)$, then $\beta_{n}(S)<\beta_{n}(T)$.

Proof. Suppose that $\omega(S) \subseteq \omega(T)$. It is easy to check that if $\mu$ is a $c d$ monomial such that the expansion of $\mu(a+b, a b+b a)$ contains the term $u_{T}$ (necessarily with coefficient 1 ), then it also contains the term $u_{S}$. Since $\Phi_{n}(c, d)$ has nonnegative coefficients, it follows that $\beta_{n}(S) \leq \beta_{n}(T)$.

Now assume that $S$ and $T$ are any subsets of $[n-1]$ for which $\omega(S) \subset \omega(T)$ (strict containment). We can easily find a $c d$-monomial $\Phi_{w}$ for which $\omega(T) \supseteq \omega\left(S_{w}\right)$ but $\omega(S) \nsupseteq \omega\left(S_{w}\right)$. For instance, if $i \in \omega(T)-\omega(S)$ then let $\Phi_{w}=c^{i-1} d c^{n-2-i}$, so $\omega\left(S_{w}\right)=\{i\}$. It follows that $\beta_{n}(S)<\beta_{n}(T)$.

Corollary 6.5. Let $S \subseteq[n-1]$. Then $\beta_{n}(S) \leq E_{n}$, with equality if and only if $S=\{1,3,5, \ldots\} \cap[n-1]$ or $S=\{2,4,6, \ldots\} \cap[n-1]$.

Proof. Immediate from Proposition 6.4 and equation (6.4).

Corollary 6.5 can be rephrased as follows. If we pick a permutation $w \in \mathfrak{S}_{n}$ at random (uniformly) and must predict its descent set, then it is best to bet either that $w$ is alternating or is reverse alternating. By equation (1.10) the probability of success will be about $2(2 / \pi)^{n+1} \approx 2(0.6366 \cdots)^{n+1}$.

An interesting generalization of Corollary 6.5 is due to K. Saito [62, Thm. 3.2]. Let $T$ be a tree on an $n$-element vertex set $V$. Let $\lambda$ be a labeling of $V$ with the numbers $1,2, \ldots, n$, and let $\mathfrak{o}_{\lambda}$ be the orientation of (the edges of) $T$ obtained by orienting $u \rightarrow v$ if $u v$ is an edge of $T$ with $\lambda(u)<\lambda(v)$. For each orientation $\mathfrak{o}$ of $T$, let $\beta(\mathfrak{o})$ be the number of labelings $\lambda$ for which $\mathfrak{o}=\mathfrak{o}_{\lambda}$.

Proposition 6.6. The orientations o that maximize $\beta(\mathfrak{o})$ are the two "bipartite orientations," i.e., those containing no directed path of length 2. 
Corollary 6.5 is equivalent to the special case of Proposition 6.6 for which $T$ is a path. The labelled trees that produce bipartite orientations are known as alternating or intransitive trees. They first appeared in the work of Gelfand, Graev, and Postnikov [33] in connection with the theory of hypergeometric functions. They were enumerated by Postnikov [56] and are also connected with counting regions of certain hyperplane arrangements $[\mathbf{5 7}, \S 8.1]$.

ACKNOWLEDGMENT. I am grateful to Joel Lewis for his careful proofreading of this paper.

\section{References}

1. D. André, Développement de $\sec x$ and tg $x$, C. R. Math. Acad. Sci. Paris 88 (1879), 965-979.

2. G. E. Andrews, Ramanujan's "Lost" Notebook. 1. Partial $\theta$-functions, Advances in Math. 41 (1981), 137-172.

3. V. I. Arnold, Bernoulli-Euler updown numbers associated with function singularities, their combinatorics and arithmetics, Duke Math. J. 63 (1991), 537-555.

4. V. I. Arnold, Springer numbers and morsification spaces, J. Algebraic Geometry 1 (1992), 197-214.

5. J. Baik, P. Deift, and K. Johansson, On the distribution of the length of the longest increasing subsequence of random permutations, J. Amer. Math. Soc. 12 (1999), 1119-1178.

6. W. Baldoni and M. Vergne, Kostant partitions functions and flow polytopes, Transform. Groups 13 (2008), 447-469.

7. Y. Baryshnikov and D. Romik, Enumeration formulas for Young tableaux in a diagonal strip, Israel J. Math., to appear.

8. B. C. Berndt, Ramanujan's Notebooks, Part V, Springer, New York, 1998.

9. M. Bóna, Combinatorics of Permutations. Chapman \& Hall/CRC, Boca Raton, FL, 2004.

10. M. Bóna, private communication dated October 13, 2005.

11. N. G. de Bruijn, Permutations with given ups and downs, Nieuw Arch. Wisk. 18 (1970), 61-65.

12. A. R. Calderbank, P. Hanlon, and R. W. Robinson, Partitions into even and odd block size and some unusual characters of the symmetric groups, Proc. London Math. Soc. 53 (1986), $288-320$.

13. D. Chebikin and R. Ehrenborg, The $f$-vector of the descent polytope, preprint; arXiv: 0812/1249.

14. C.-O. Chow and W. C. Shiu, Counting simsun permutations by descents, Ann. Combinatorics, to appear.

15. R. Cori, S. Dulucq, and X. G. Viennot, Shuffle of parenthesis systems and Baxter permutations, J. Combin. Theory Ser. A 43 (1986), 1-22.

16. E. Deutsch and S. Elizalde, Restricted simsun permutations, preprint; arXiv:0912.1361.

17. E. E. Doberkat, Problem 84-20, SIAM Review 26 (1984), 580.

18. R. Donaghey, Alternating permutations and binary increasing trees, J. Combin. Theory Ser. A 18 (1975), 141-148.

19. S. Dulucq and R. Simion, Combinatorial statistics on alternating permutations, J. Algebraic Combinatorics 8 (1998), 169-191.

20. R. Ehrenborg and S. Mahajan, Maximizing the descent statistic, Ann. Comb. 2 (1998), 111129.

21. N. Elkies, On the sums $\sum_{k=-\infty}^{\infty}(4 k+1)^{-n}$, Amer. Math. Monthly 110 (2003), 561-573.

22. R. C. Entringer, A combinatorial interpretation of the Euler and Bernoulli numbers, Nieuw. Arch. Wisk. 14 (1966), 241-246.

23. P. Flajolet and R. Sedgewick, Analytic Combinatorics, Cambridge University Press, Cambridge, 2009.

24. D. Foata, Groupes de réarrangements et nombres d'Euler, C. R. Acad. Sci. Paris Sér. A-B 275 (1972), A1147-A1150.

25. D. Foata and G.-N. Han, Doubloons and new $q$-tangent numbers, preprint; www-irma.u-strasbg.fr/ foata/paper/pub111doubloon.pdf. 
26. D. Foata and M.-P. Schützenberger, Nombres d'Euler et permutations alternantes, in A Survey of Combinatorial Theory (Proc. Internat. Sympos., Colorado State Univ., Fort Collins, Colo., 1971), North-Holland, Amsterdam, 1973, pp. 173-187.

27. D. Foata and V. Strehl, Rearrangements of the symmetric group and enumerative properties of the tangent and secant numbers, Math. Z. 137 (1974), 257-264.

28. H. O. Foulkes, Enumeration of permutations with prescribed up-down and inversion sequences, Discrete Math. 15 (1976), 235-252.

29. H. O. Foulkes, Tangent and secant numbers and representations of symmetric groups, Discrete Math.

30. J. Françon and G. Viennot, Permutations selon leurs pics, creux, doubles montées et double descentes, nombres d'Euler et nombres de Genocchi, Discrete Math. 28 (1979), 21-35.

31. M. Fulmek, A continued fraction expansion for a $q$-tangent function, Sém. Lothar. Combin. B45b (2000), 3 pp.

32. W. F. Galway, An asymptotic expansion of Ramanujan, in Number Theory, Fifth Conference of the Canadian Number Theory Association (R. Gupta and K. S. Williams, eds.), CRM Proceedings and Lecture Notes 19, American Mathematical Society, Providence, RI, 1999, pp. $107-110$

33. I. M. Gelfand, M. I. Graev, and A. Postnikov, Hypergeometric functions associated with positive roots, in Arnold-Gelfand Mathematical Seminars: Geometry and Singularity Theory (V. I. Arnold, I. M. Gelfand, et al., eds.), Birkhäuser, Boston, 1996, pp. 205-221.

34. I. M. Gessel, Symmetric functions and P-recursiveness. J. Combin. Theory Ser. A 53 (1990), $257-285$.

35. J. Ginsburg, Stirling numbers, Encyclopedia Britannica, 1965.

36. I. P. Goulden and D. M. Jackson, Algebraic methods for permutations with prescribed patterns, Adv. in Math. 42 (1981), 113-135.

37. I. P. Goulden and D. M. Jackson, Combinatorial Enumeration, Wiley-Interscience Series in Discrete Mathematics, John Wiley \& Sons, New York, 1983; reprinted by Dover, Minneola, NY, 2004.

38. R. L. Graham, D. E. Knuth, and O. Patashnik, Concrete Mathematics, second ed., AddisonWesley, Reading, MA, 1994.

39. O. Guibert and S. Linusson, Doubly alternating Baxter permutations are Catalan, Discrete Math. 217 (2000), 157-166.

40. G.-N. Han, A. Randrianarivony, and J. Zeng, Un autre $q$-analogue des nombres d'Euler, in The Andrews Festschrift. Seventeen Papers on Classical Number Theory and Combinatorics (D. Foata and G.-N. Han, eds.), Springer-Verlag, Berlin/Heidelberg, 2001, pp. 139-158; Sém. Lothar. Combin. B42e, 22 pp.

41. G. Hetyei and E. Reiner, Permutation trees and variation statistics, European J. Combin. 19 (1998), 847-866

42. G. Hong, Catalan numbers in pattern-avoiding permutations, MIT Undergraduate J. Math. 10 (2008), 53-68.

43. M. Josuat-Vergès, A q-enumeration of alternating permutations, European J. Combin., to appear.

44. A. J. Kempner, On the shape of polynomial curves, Tôhoku Math. J. 37 (1933), 347-362.

45. J. B. Lewis, Alternating, pattern-avoiding permutations, Electronic J. Combinatorics 16 (2009), N7.

46. A. G. Kuznetsov, I. M. Pak, and A. E. Postnikov, Increasing trees and alternating permutations, Russian Math. Surveys 49:6 (1994), 79-114; translated from Uspekhi Mat. Nauk 49:6 (1994), 79-110.

47. J. B. Lewis, Pattern avoidance and RSK-like algorithms for alternating permutations and Young tableaux, preprint.

48. B. F. Logan and L. A. Shepp, A variational problem for random Young tableaux. Advances in Math. 26 (1977), 206-222.

49. I. G. Macdonald, Symmetric Functions and Hall Polynomials, second ed., Oxford University Press, Oxford, 1995.

50. T. Mansour, Restricted 132-alternating permutations and Chebyshev polynomials, Ann. Combinatorics 7 (2003), 201-227.

51. J. Millar, N. J. A. Sloane, and N. E. Young, A new operation on sequences: the boustrophedon transform, J. Combinatorial Theory, Ser. A 76 (1996), 44-54. 
52. S. Min and S. Park, The enumeration of doubly alternating Baxter permutations, J. Korean Math. Soc. 43 (2006), 553-561.

53. I. Niven, A combinatorial problem of finite sequences, Nieuw Arch. Wisk. 16 (1968), 116-123.

54. E. Ouchterlony, Pattern avoiding doubly alternating permutations, Proc. FPSAC 2006, garsia.math. yorku.ca/fpsac06/papers/83.pdf.

55. R. Pemantle and M. C. Wilson, Asymptotics of multivariate sequences. I. Smooth points of the singular variety, J. Combin. Theory Ser. A 97 (2002), 129-161.

56. A. Postnikov, Intransitive trees, J. Combin. Theory Ser. A. 79 (1997), 360-366.

57. A. Postnikov and R. Stanley, Deformations of Coxeter hyperplane arrangements, J. Combinatorial Theory (A) 91 (2000), 544-597.

58. H. Prodinger, Combinatorics of geometrically distributed random variables: new $q$-tangent and $q$-secant numbers, Int. J. Math. Math. Sci. 24 (2000), 825-838.

59. H. Prodinger, A continued fraction expansion for a $q$-tangent function: an elementary proof, Sém. Lothar. Combin. B60b (2008), 3 pp.

60. F. J. Rispoli, Fibonacci polytopes and their applications, Fibonacci Quart. 43 (2005), 227233.

61. G.-C. Rota and B. Taylor, The classical umbral calculus, SIAM J. Math. Anal. 25 (1994), 694-711.

62. K. Saito, Principal Г-cone for a tree. Adv. Math. 212 (2007), 645-668.

63. L. Seidel, Über eine einfache Entstehungsweise der Bernoullischen Zahlen und einiger verwandten Reihen, Sitzungsber. Münch. Akad. 4 (1877), 157-187.

64. R. Stanley, Binomial posets, Möbius inversion, and permutation enumeration, J. Combinatorial Theory (A) $\mathbf{2 0}$ (1976), 336-356.

65. R. Stanley, Exponential structures, Studies in Applied Math. 59 (1978), 73-82.

66. R. Stanley, Elementary problem E 2701, American Math. Monthly 85 (1978), 197; solution by I. G. Macdonald and R. B. Nelsen (independently), 86 (1979), 396.

67. R. Stanley, Some aspects of groups acting on finite posets, J. Combin. Theory Ser. A 32 (1982), 132-161.

68. R. Stanley, Two poset polytopes, Discrete Comput. Geom. 1 (1986), 9-23.

69. R. P. Stanley, Flag $f$-vectors and the $c d$-index, Math. Z. 216 (1994), 483-499.

70. R. Stanley, Enumerative Combinatorics, vol. 1, Wadsworth and Brooks/Cole, Pacific Grove, CA, 1986; second printing, Cambridge University Press, New York/Cambridge, 1996.

71. R. Stanley, Enumerative Combinatorics, vol. 1, second edition, in preparation; Chapter 1 available at math.mit.edu/ rstan/ec/ch1.pdf.

72. R. Stanley, Enumerative Combinatorics, vol. 2, Cambridge University Press, New York/Cambridge, 1999.

73. R. Stanley, Increasing and decreasing subsequences and their variants, Proc. Internat. Cong. Math. (Madrid, 2006), American Mathematical Society, Providence, RI, 2007, pp. 545-579.

74. R. Stanley, Alternating permutations and symmetric functions, J. Combinatorial Theory (A) 114 (2007), 436-460.

75. R. Stanley, Longest alternating subsequences of permutations, Michigan Math. J. 57 (2008), 675-687.

76. R. Stanley, Catalan addendum, math.mit.edu/ rstan/ec/catadd.pdf.

77. S. Sundaram, The homology representations of the symmetric group on Cohen-Macaulay subposets of the partition lattice, Adv. Math. 104 (1994), 225-296.

78. G. Sylvester, Continuous spin ising ferromagnets, Ph.D. thesis, M.I.T., 1976.

79. C. A. Tracy, and H. Widom, Level-spacing distributions and the Airy kernel, Comm. Math. Phys. 159 (1994), 151-174.

80. A. M. Vershik and S. V. Kerov, Asymptotic behavior of the Plancherel measure of the symmetric group and the limit form of Young tableaux (Russian). Dokl. Akad. Nauk SSSR 223 (1977), 1024-1027. English translation: Soviet Math. Dokl. 233 (1977), 527-531.

81. G. Viennot, Permutations ayant une forme donnée, Discrete Math. 26 (1979), 279-284.

82. G. Viennot, Interprétations combinatoires des nombres d'Euler et de Genocchi, Seminar on Number Theory, 1981/1982, No. 11, Univ. Bordeaux I, Talence, 1982.

83. H. Widom, On the limiting distribution for the longest alternating subsequence in a random permutation, Electron. J. Combin. 13(1) (2006), Article R25.

84. H. S. Wilf, Real zeroes of polynomials that count runs and descending runs, preprint, 1998. 
85. D. Zeilberger, Proof of a conjecture of Chan, Robbins, and Yuen, Electron. Trans. Numer. Anal. 9 (1999), 147-148.

Department of Mathematics, M.I.T., Cambridge, MA 02139

E-mail address: rstan@math.mit.edu 